\title{
Schoen manifold with line bundles as resolved magnetized orbifolds
}

\author{
Stefan Groot Nibbelink ${ }^{a}$ and Patrick K.S. Vaudrevange ${ }^{b}$ \\ ${ }^{a}$ Arnold Sommerfeld Center for Theoretical Physics, \\ Ludwig-Maximilians-Universität München, 80333 München, Germany \\ ${ }^{b}$ Deutsches Elektronen-Synchrotron DESY, \\ Notkestraße 85, 22607 Hamburg, Germany \\ E-mail: Groot.Nibbelink@physik.uni-muenchen.de, \\ patrick.vaudrevange@desy.de
}

ABSTRACT: We give an alternative description of the Schoen manifold as the blow-up of a $\mathbb{Z}_{2} \times \mathbb{Z}_{2}$ orbifold in which one $\mathbb{Z}_{2}$ factor acts as a roto-translation. Since for this orbifold the fixed tori are only identified in pairs but not orbifolded, four-dimensional chirality can never be obtained in heterotic string compactifications using standard techniques alone. However, chirality is recovered when its tori become magnetized. To exemplify this, we construct an $\mathrm{E}_{8} \times \mathrm{E}_{8}{ }^{\prime}$ heterotic SU(5) GUT on the Schoen manifold with Abelian gauge fluxes, which becomes an MSSM with three generations after an appropriate Wilson line is associated to its freely acting involution. We reproduce this model as a standard heterotic orbifold CFT of the (partially) blown down Schoen manifold with a magnetic flux. Finally, in analogy to a proposal for non-perturbative heterotic models by Aldazabal et al. we suggest modifications to the heterotic orbifold spectrum formulae in the presence of magnetized tori.

Keywords: Flux compactifications, Superstrings and Heterotic Strings, Conformal Field Models in String Theory

ARXIV EPRINT: 1212.4033 


\section{Contents}

1 Introduction and summary 1

2 Heterotic $\mathbb{Z}_{2} \times \mathbb{Z}_{2}$ orbifolds $\quad 4$

2.1 General features of $\mathbb{Z}_{2} \times \mathbb{Z}_{2}$ orbifolds 4

2.2 The standard DW(0-1) $\mathbb{Z}_{2} \times \mathbb{Z}_{2}$ orbifold 6

2.3 The $\mathrm{DW}(0-2) \mathbb{Z}_{2} \times \mathbb{Z}_{2}$ orbifold 7

3 Schoen manifold $\quad 9$

3.1 Singular Schoen manifold: the DW(0-2) orbifold 10

3.2 Schoen manifold as the resolution of the DW(0-2) orbifold 10

4 Line bundle models on the Schoen resolution $\quad 12$

$\begin{array}{ll}4.1 \text { Abelian gauge flux backgrounds } & 12\end{array}$

$\begin{array}{lll}4.2 & \text { Spectra computation } & 15\end{array}$

$\begin{array}{lll}4.3 & \text { Interpretation as blow-up of DW(0-2) orbifold models } & 17\end{array}$

$\begin{array}{ll}4.4 \text { Sample model: an eight generation GUT } & 18\end{array}$

5 A line bundle MSSM on the Schoen manifold 20

5.1 Six GUT generations on the Schoen resolution 20

$\begin{array}{lll}5.2 & \text { Freely acting } \mathbb{Z}_{2} \text { and MSSM with three generations } & 21\end{array}$

5.3 Singular limits of the Schoen GUT with line bundles 22

5.4 Schoen line bundle MSSM as a blown up orbifold 23

6 Towards an CFT description of orbifolds with magnetized tori $\quad 27$

$\begin{array}{lll}\text { 6.1 Standard modular invariance conditions } & 27\end{array}$

6.2 Heterotic description of the Schoen orbifold with magnetized tori 28

6.3 Sample model as blow-up of orbifold with magnetized tori 29

\section{Introduction and summary}

There are two standard approaches in the literature to geometrical string compactification of the heterotic string. (Non-geometrical approaches involve e.g. free-fermionic models $[1$, $2]$ and Gepner constructions [3,4].) Either strings are considered on singular (toroidal) orbifolds or on smooth Calabi-Yau manifolds. The main advantage of orbifolds over smooth Calabi-Yau spaces is that they are so simple that the heterotic string can be quantized on them exactly $[5,6]$. Therefore, one has access to the full spectrum of the theory; not just to its zero modes. In addition, one can scan in a very systematic way through the parameter space of heterotic orbifold compactifications in order to search for interesting models for 
string phenomenology (using e.g. [7]). This has resulted, for example, in a mini-landscape of a few hundred MSSM models based on the heterotic $\mathrm{E}_{8} \times \mathrm{E}_{8}{ }^{\prime}$ orbifold $T^{6} / \mathbb{Z}_{6-\mathrm{II}}[8,9]$.

An orbifold can be considered as a Calabi-Yau space at a singular point in its moduli space where symmetries get enhanced. To go away from the orbifold point in moduli space the orbifold singularities have to be resolved (or deformed). In this blow-up process certain (exceptional) cycles that were hidden inside the singularities acquire finite volumes. From the heterotic orbifold model perspective this corresponds to turning on Vacuum-Expectation-Values (VEVs) for twisted states, so-called blow-up modes, which are localized at the singularities of the orbifold. Unfortunately, an exact string quantization is out of reach at a generic point in moduli space and there is typically much less symmetry. For example, it turns out that in full blow-up any mini-landscape model has broken hypercharge $[10,11]$. This might be interpreted in two ways: Either one does not go to the full blow-up in order to keep hypercharge unbroken and our string vacuum is very close to the orbifold point, or our string vacuum is at a generic point of the moduli space and different constructions are needed for phenomenology. As discussed in [12, 13] freely acting involutions can be used as an example for the second interpretation and an MSSM orbifold model has been constructed on the heterotic $T^{6} / \mathbb{Z}_{2} \times \mathbb{Z}_{2}$ orbifold, which in principle can avoid hypercharge breaking in full blow-up.

Furthermore, there have been various constructions of MSSM models in the context of the heterotic string compactified on smooth Calabi-Yau manifolds. For example, a three generation MSSM has been constructed in [14] on the Schoen manifold [15] using a stable $\mathrm{SU}(5)$ vector bundle [16-18]. Similar constructions — yet not fully supersymmetric [19] can be found in e.g. [20]. Even though the Schoen manifold is just one particular CalabiYau space, it is a typical example of a complete intersection Calabi-Yau: It can be obtained as a set of hyper surfaces within a direct product of projective spaces.

Most heterotic models built on the Schoen manifold require complicated constructions of stable $\mathrm{SU}(\mathrm{N})$ bundles. Therefore, one may wonder whether it is also possible to design MSSM-like heterotic string models on the Schoen manifolds using line bundles. As has been realized by various groups [21, 22] the analysis of line bundles on smooth Calabi-Yau spaces, described as complete intersections in toric varieties, can be performed much easier than their non-Abelian counterparts. The main reason for this is that for line bundle gauge backgrounds the stability of the bundle reduces to solving simple Donaldson-Uhlenbeck-Yau (DUY) equations [23, 24] in terms of the Kähler moduli [25, 26]. Moreover, the embedding of line bundles into the ten-dimensional heterotic gauge group $\left(\mathrm{E}_{8} \times \mathrm{E}_{8}{ }^{\prime}\right.$ or $\mathrm{SO}(32)$, where we focus on the $\mathrm{E}_{8} \times \mathrm{E}_{8}{ }^{\prime}$ case, but most of our results equally apply to the $\mathrm{SO}(32)$ case.) can be characterized by vectors of integers $[27,28]$. This makes it possible to perform computer-aided scans for potentially phenomenologically viable models.

The Schoen manifold does not only provide an interesting example of a Calabi-Yau constructed as a complete intersection. It can also be considered as a smooth limit of a certain orbifold [29]. This orbifold has some special properties: It is a $T^{6} / \mathbb{Z}_{2} \times \mathbb{Z}_{2 \text {,rototrans }}$

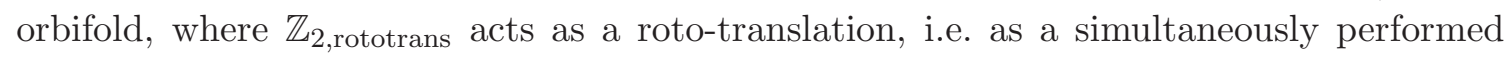
rotation and translation [30] (and [31] in the type II string context, where this kind of orbifolds are called shift orbifolds). This has far reaching consequences for the structure 
of the fixed points and tori and, in turn, modifies the breaking of higher dimensional supersymmetry to $\mathcal{N}=1$ in four dimensions for heterotic string compactifications. As we will see, this necessarily results in vector-like spectra for this kind of orbifold geometry.

This is not a peculiar feature of this special orbifold, many more orbifolds with this property are known. Recently, there has been a classification of all six-dimensional toroidal orbifold geometries that give rise to four-dimensional $\mathcal{N}=1$ supersymmetry [32]. These geometries can be arranged in two sets: The ones with Abelian point group and the ones with non-Abelian point group. 23 of the 138 geometries with Abelian point group share the property that they necessarily lead to non-chiral spectra for heterotic string compactifications. (These are $\mathbb{Z}_{2} \times \mathbb{Z}_{2}$ variants, part of the classification of ref. [29].) For the non-Abelian cases these numbers are essentially unknown. However, one explicit example of a heterotic $S_{3}$ orbifold [33] also turns out to produce only vector-like spectra. We therefore expect that also a sizable portion of the non-Abelian point group orbifolds will unavoidably be non-chiral in four dimensions.

Hence, it is an important question whether there exists an unavoidable no-go theorem against four-dimensional chirality for all these heterotic orbifolds. Fortunately, we will show that it is possible to circumvent this no-go by allowing for magnetized tori on the orbifold. Concretely, we put magnetic fluxes on the tori of the Schoen orbifold and show that four-dimensional chiral spectra can be realized. More than that, we will show that it is even possible to obtain MSSM-like models in this way.

There is one technical subtlety in the construction of such orbifolds with magnetized tori: As far as we know, contrary to conventional orbifolds, it is unknown how to quantize the heterotic string exactly on them. We by-pass this obstruction in two ways: First, we consider the whole construction in blow-up, i.e. on the smooth Schoen manifold. Second, we show that one can start with a six-dimensional spectrum obtained from a standard heterotic $T^{4} / \mathbb{Z}_{2}$ orbifold, which is a subspace of the partially blown-down Schoen manifold, using conventional CFT techniques. Then, one can use field theoretical methods, discussed e.g. in [34-36], to determine the consequences of the additional (magnetic) fluxes and to obtain a chiral spectrum in four dimensions. Both approaches, i.e. the smooth approach and the hybrid approach of combining CFT and field theoretical methods, will reproduce exactly the same spectrum.

Paper overview. In section 2 we review the basics of heterotic orbifold models. In addition we introduce the DW(0-2) orbifold which is of central interest in this work. Section 3 provides an alternative description of the Schoen manifold as the resolution of this DW(0-2) orbifold. In section 4 we describe the $\mathrm{E}_{8} \times \mathrm{E}_{8}{ }^{\prime}$ heterotic string with line bundles on the divisors of the Schoen manifold including magnetic fluxes on the tori of the underlying orbifold. Moreover, we identify the relevant consistency conditions for such gauge backgrounds and compute the resulting chiral spectra in both, four and six, dimensions. Then, we provide an example that mainly serves to illustrate various aspects of the general theory developed in this paper. In section 5 we construct a specific example, which is potentially phenomenologically interesting as it has the particle spectrum of the MSSM in four dimensions. We analyze this example using two approaches: First, the smooth ap- 
proach and, second, the hybrid approach of combining CFT and field theoretical methods. Finally, in section 6 we speculate on how to extend the standard heterotic CFT description of orbifolds in the presence of magnetized tori.

\section{Acknowledgments}

We would like to thank Vincent Bouchard and Ron Donagi for early discussions that initiated this project. We are also indebted to Kang-Sin Choi, James Gray, Tatsuo Kobayashi and Fabian Rühle for valuable discussions. SGN would like to thank the organizers of the Workshop "Topological Heterotic Strings and (0,2) Mirror Symmetry" in Vienna for hospitality. We also thank the organizers of the Bethe Forum and the 4th Bethe Center Workshop on "Unification and String Theory" in Bonn/Bad Honnef for hospitality. This research has been supported by the "LMUExcellent" Programme. P.V. is supported by SFB grant 676.

\section{Heterotic $\mathbb{Z}_{2} \times \mathbb{Z}_{2}$ orbifolds}

In this section we describe some basic geometrical properties of $\mathbb{Z}_{2} \times \mathbb{Z}_{2}$ orbifolds and explain how to determine whether such orbifolds can lead to heterotic $\left(\mathrm{E}_{8} \times \mathrm{E}_{8}{ }^{\prime}\right)$ string models with chiral spectra in four dimensions. We follow the classification scheme for these orbifolds developed by Donagi-Wendland [29]. (See their table 1 for details and nomenclature). In particular, we describe their DW(0-2) orbifold which can be considered as a certain singular limit of the so-called Schoen manifold. However, for comparison purposes we first recall some basic facts of $\mathbb{Z}_{2} \times \mathbb{Z}_{2}$ orbifolds and give some details of the more often considered DW(0-1) orbifold.

\subsection{General features of $\mathbb{Z}_{2} \times \mathbb{Z}_{2}$ orbifolds}

We consider $\mathbb{Z}_{2} \times \mathbb{Z}_{2}$ orbifolds defined as

$$
\mathbb{R}^{6} / S
$$

where the space group $S$ specifies an equivalence relation on $\mathbb{R}^{6}$ as $g X \sim X$ for all $g \in S$ and $X \in \mathbb{R}^{6}$. A general space group element $g=(\vartheta, \ell)$ consists of a six-dimensional rotation matrix $\vartheta$ and a translation $\ell$. It acts on $X \in \mathbb{R}^{6}$ as $g X=\vartheta X+\ell$. The space group is generated by two types of elements: The purely translational elements $g_{i}=\left(\mathbb{1}, e_{i}\right)$ are determined by six basis vectors $e_{i}(i=1, \ldots, 6)$ that span a six-dimensional lattice and hence define a six-torus. For simplicity, we identify $\mathbb{R}^{6}=\mathbb{C}^{3}$ and take as basis vectors

$$
\begin{array}{lll}
e_{1}=(1,0,0), & e_{2}=(i, 0,0), & e_{3}=(0,1,0), \\
e_{4}=(0, i, 0), & e_{5}=(0,0,1), & e_{6}=(0,0, i) .
\end{array}
$$

Consequently, we denote the torus coordinates by $z=\left(z_{1}, z_{2}, z_{3}\right) \in T_{1}^{2} \times T_{2}^{2} \times T_{3}^{2}$ in this complex basis. The remaining two generators of the space group, $g_{\theta}$ and $g_{\omega}$, involve $\mathbb{Z}_{2} \times \mathbb{Z}_{2}$ rotations, denoted by $\theta$ and $\omega$, possibly combined with some translations. When this is the 
case such elements are referred to as roto-translations. The phases of the rotations acting on $\mathbb{C}^{3}$ are

$$
v_{\theta}=\left(0, \frac{1}{2},-\frac{1}{2}\right), \quad \text { and } \quad v_{\omega}=\left(-\frac{1}{2}, 0, \frac{1}{2}\right)
$$

respectively.

The action of the space group elements is subsequently extended to the left-moving sector of the heterotic worldsheet theory that describes the target space gauge degrees of freedom. In a bosonic formulation this sector can be described by 16 left-moving coordinates $X_{L}^{I}(I=1, \ldots, 16)$ living on a torus $\mathbb{R}^{16} / \Lambda_{\mathrm{E}_{8} \times \mathrm{E}_{8}{ }^{\prime}}$ defined by the $\mathrm{E}_{8} \times \mathrm{E}_{8}{ }^{\prime}$ root lattice $\Lambda_{\mathrm{E}_{8} \times \mathrm{E}_{8}{ }^{\prime}}$. The simplest way to extend the space group action is the shift embedding which acts as: $g X_{L}^{I}=X_{L}^{I}+2 \pi V_{g}^{I}$. Hence $V: g \mapsto V_{g}$ defines a group homomorphism of the space group $S$ to the Abelian group $\mathbb{R}^{16}$ under addition. For a general space group element $g=g_{\theta}^{k} g_{\omega}^{l} g_{1}^{n_{1}} \cdot \ldots \cdot g_{6}^{n_{6}}$, with $k, l=0,1$ and $n_{i} \in \mathbb{Z}$, the local twist $v_{g}$ and shift vector $V_{g}$ can be expanded as

$$
v_{g}=k v_{\theta}+l v_{\omega}, \quad V_{g}=k V_{\theta}+l V_{\omega}+n_{i} W_{i}
$$

in terms of the gauge shift vectors $V_{\theta}$ and $V_{\omega}$ and the discrete Wilson lines $W_{i}$ where summation over $i$ from 1 to 6 is understood. In order that $V_{g}$ defines a proper group homomorphism, it is required that

$$
2 V_{\theta} \cong 2 V_{\omega} \cong 2 W_{i} \cong 0
$$

where $\cong$ means equal up to $\Lambda_{\mathrm{E}_{8} \times \mathrm{E}_{8}}$ lattice vectors.

The central consistency requirement of heterotic orbifold compactifications is modular invariance. For a $\mathbb{Z}_{2} \times \mathbb{Z}_{2}$ orbifold it requires for all commuting space group elements $h, g$ that

$$
V_{h} \cdot V_{g}-v_{h} \cdot v_{g} \equiv 0
$$

where $\equiv$ indicates that both sides are equal up to integers. Combined with equation (2.5) this leads to the following set of irreducible modular invariance conditions:

$$
V_{\theta}^{2} \equiv v_{\theta}^{2}, \quad V_{\omega}^{2} \equiv v_{\omega}^{2}, \quad V_{\theta} \cdot V_{\omega} \equiv v_{\theta} \cdot v_{\omega}, \quad V_{\theta} \cdot W_{i} \equiv V_{\omega} \cdot W_{i} \equiv 0, \quad W_{i} \cdot W_{j} \equiv 0,
$$

by going through all possible commuting choices of $g, h \in S$.

The spectrum of (twisted or untwisted) closed strings from the sector $g \in S$ is dictated by their left- and right-moving masses

$$
M_{L}^{2}=\frac{1}{2} P_{\mathrm{sh}}^{2}+\tilde{N}_{g}-\frac{3}{4}, \quad M_{R}^{2}=\frac{1}{2} p_{\mathrm{sh}}^{2}-\frac{1}{4},
$$

in terms of the (shifted) left- and right-moving momenta

$$
P_{\mathrm{sh}}=P+V_{g}, \quad p_{\mathrm{sh}}=p+v_{g},
$$




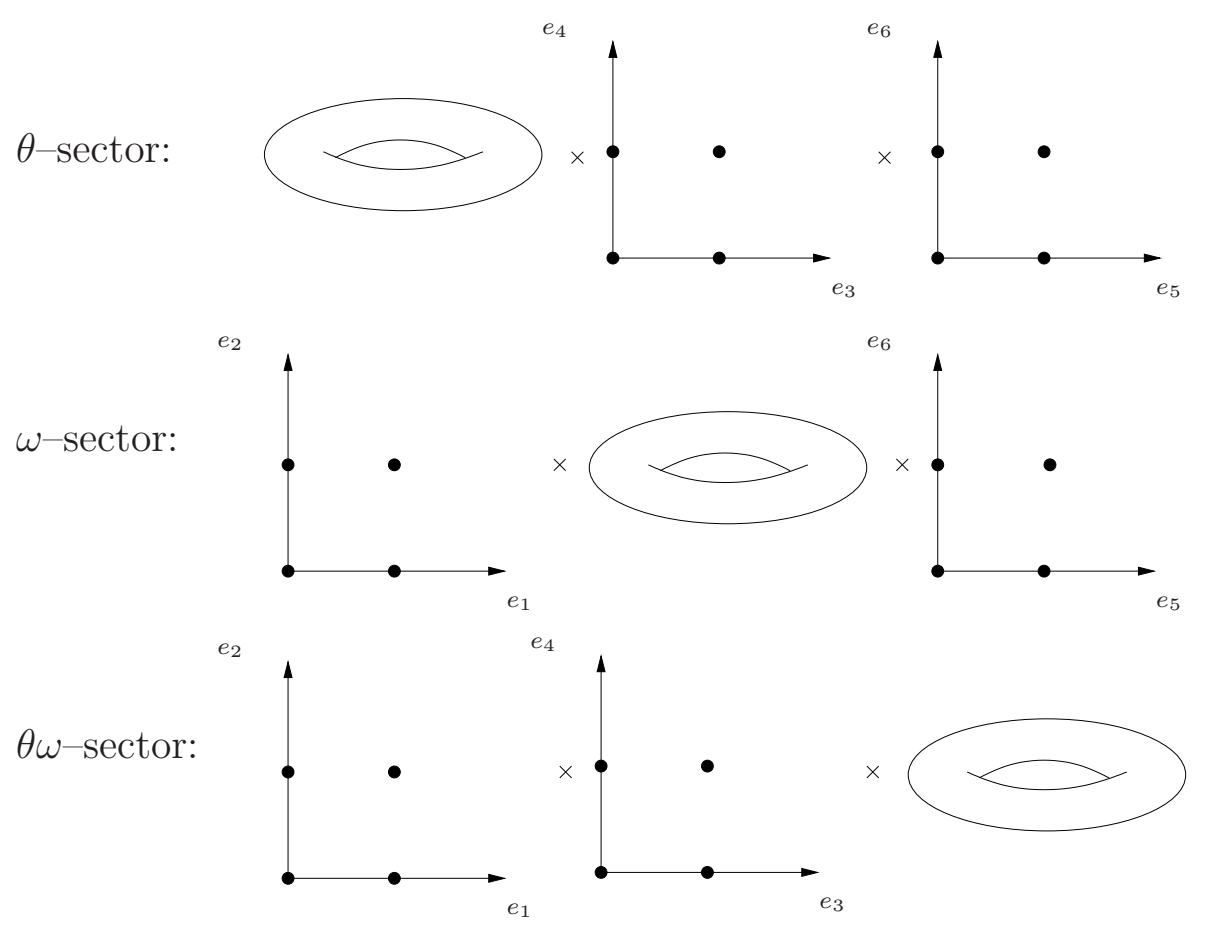

Figure 1. Fixed tori of the DW(0-1) orbifold. Every fixed torus intersects $4+4$ other fixed tori and the intersection loci are points in six dimensions.

where $P \in \Lambda_{\mathrm{E}_{8} \times \mathrm{E}_{8}{ }^{\prime}}$ and $p$ is from the vectorial or spinorial weight lattice of $\mathrm{SO}(8)$. Here, the twist vector $v_{g}$ is extended to a four-dimensional vector with an extra 0 as first component. Furthermore, $\widetilde{N}_{g}$ is a (fractional or integer) number operator counting the number of leftmoving oscillators $\tilde{\alpha}_{-n}$ acting on the left-moving ground state of the $g$-twisted sector. The physical spectrum is subject to the level matching condition $M_{L}^{2}=M_{R}^{2}$. The massless states in four dimensions have vanishing left- and right-moving masses, $M_{L}=M_{R}=0$, and are subject to the projection conditions

$$
V_{h} \cdot P_{\mathrm{sh}}-v_{h} \cdot\left(p_{\mathrm{sh}}+\Delta \tilde{N}_{g}\right) \equiv \frac{1}{2}\left(V_{g} \cdot V_{h}-v_{g} \cdot v_{h}\right)
$$

for all space group elements $h$ that commute with $g$, using $\Delta \tilde{N}_{g}^{i}=\tilde{N}_{g}^{\bar{i}}-\tilde{N}_{g}^{i}, i=0,1,2,3$, where $\tilde{N}_{g}^{\bar{i}}$ and $\tilde{N}_{g}^{i}$ are integer oscillator numbers counting the numbers of oscillators $\tilde{\alpha}_{-n}^{\bar{i}}$ and $\tilde{\alpha}_{-n}^{i}$ acting on the ground state of the $g$-twisted sector, respectively.

\subsection{The standard DW(0-1) $\mathbb{Z}_{2} \times \mathbb{Z}_{2}$ orbifold}

Now, we consider the standard $T^{6} / \mathbb{Z}_{2} \times \mathbb{Z}_{2}$ orbifold which corresponds to the $\operatorname{DW}(0-1)$ model of ref. [29] in order to see how four-dimensional chirality arises. In this case, the elements $g_{\theta}$ and $g_{\omega}$ act only as rotations, hence the space group $S$ is generated by the elements: $g_{\theta}=(\theta, 0), g_{\omega}=(\omega, 0)$ and $g_{i}=\left(\mathbb{1}, e_{i}\right)$.

When compactifying the heterotic string on this orbifold, massless strings are attached to its fixed tori. There are $16+16+16$ fixed tori associated to three twisted sectors with 


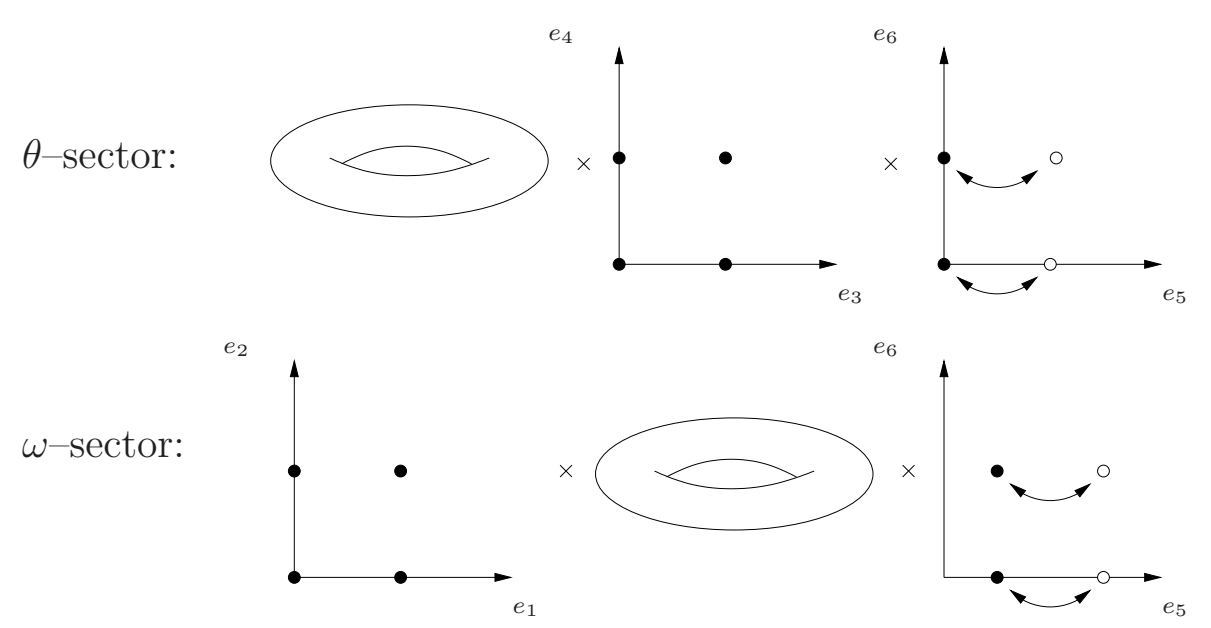

Figure 2. Fixed tori of the DW(0-2) orbifold. The fixed tori of the $\theta$-sector never intersect the ones from the $\omega$-sector, as they lie displaced in the third torus.

orbifold elements $g_{\theta}, g_{\omega}$ and $g_{\theta} g_{\omega}$. These fixed tori are in one-to-one correspondence to the space group elements:

$$
\begin{gathered}
\left(\theta, n_{i} e_{i}\right) \text { for } n_{1}=n_{2}=0 \text { and } n_{3}, n_{4}, n_{5}, n_{6}=0,1, \\
\left(\omega, n_{i} e_{i}\right) \text { for } n_{3}=n_{4}=0 \text { and } n_{1}, n_{2}, n_{5}, n_{6}=0,1, \\
\left(\theta \omega, n_{i} e_{i}\right) \text { for } n_{5}=n_{6}=0 \text { and } n_{1}, n_{2}, n_{3}, n_{4}=0,1,
\end{gathered}
$$

and are displayed in figure 1. At a given fixed torus there exists a six-dimensional $\mathcal{N}=1$ theory (i.e. $\mathcal{N}=2$ theory in four-dimensional language) with localized hypermultiplets on it. Since every fixed torus intersects other fixed tori, six-dimensional $\mathcal{N}=1$ supersymmetry is broken to $\mathcal{N}=1$ in four dimensions at the intersection points. Technically, each fixed torus is orbifolded by the action of some other non-trivial elements because the orbifold generators $g_{\theta}$ and $g_{\omega}$ commute. For example, the fixed torus of $(\theta, 0)$ is orbifolded by $(\omega, 0)$ and $(\theta \omega, 0)$. Hence, the projection conditions $(2.10)$ are active and reduce a hypermultiplet in six dimensions to a four-dimensional chiral superfield, giving chiral matter.

For example, in the orbifold standard embedding, where the twists $\theta$ and $\omega$ are embedded via the shifts $V_{\theta}=\left(0, \frac{1}{2},-\frac{1}{2}, 0^{5}\right)\left(0^{8}\right)$ and $V_{\omega}=\left(-\frac{1}{2}, 0, \frac{1}{2}, 0^{5}\right)\left(0^{8}\right)$, we obtain a theory with 51 chiral 27-plets ( 3 untwisted and $3 \cdot 16$ twisted) and 3 untwisted chiral $\overline{\mathbf{2 7}}$-plets of $\mathrm{E}_{6}$ in four dimensions. This precisely corresponds to the hodge numbers of the DW(0-1) orbifold: $\left(h_{11}, h_{21}\right)=(51,3)$.

\subsection{The DW(0-2) $\mathbb{Z}_{2} \times \mathbb{Z}_{2}$ orbifold}

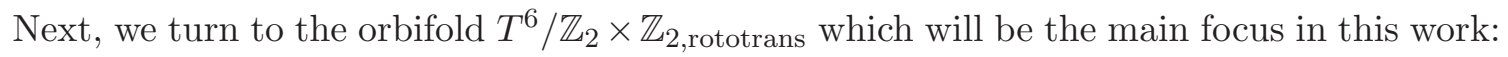
the DW(0-2) model in the classification [29]. Its space group $S$ is generated by the elements $g_{\theta}=(\theta, 0), g_{\omega}=\left(\omega, \frac{1}{2} e_{5}\right)$ and $g_{i}=\left(\mathbb{1}, e_{i}\right)$. In detail, the action of $g_{\theta}, g_{\omega}, g_{\omega} g_{\theta}$ and $g_{\theta} g_{\omega}$ 
on $\mathbb{C}^{3}$ is given by

$$
\begin{array}{ll}
g_{\theta}:\left(z_{1}, z_{2}, z_{3}\right)=\left(z_{1},-z_{2},-z_{3}\right), & g_{\omega} g_{\theta}:\left(z_{1}, z_{2}, z_{3}\right)=\left(-z_{1},-z_{2}, z_{3}+\frac{1}{2}\right), \\
g_{\omega}:\left(z_{1}, z_{2}, z_{3}\right)=\left(-z_{1}, z_{2},-z_{3}+\frac{1}{2}\right), & g_{\theta} g_{\omega}:\left(z_{1}, z_{2}, z_{3}\right)=\left(-z_{1},-z_{2}, z_{3}-\frac{1}{2}\right) .
\end{array}
$$

This shows explicitly that $g_{\theta}$ acts as an ordinary $\mathbb{Z}_{2}$ rotation, while $g_{\omega}$ defines a $\mathbb{Z}_{2}$ roto-

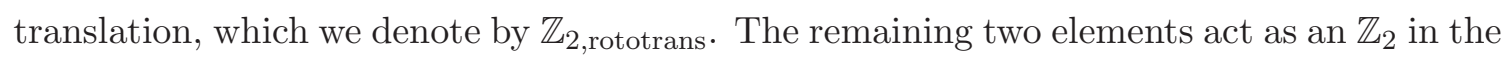
first two two-tori, but as a translation over half a lattice vector $e_{5}$ in the third two-torus.

This has various important consequences for the distribution of fixed tori within the DW(0-2) orbifold: Rather than $16+16+16$ there are only $8+8$ fixed tori. These are left fixed by the group elements $g_{\theta}$ and $g_{\omega}$. Since $g_{\theta} g_{\omega}$ and $g_{\omega} g_{\theta}$ act as a pure translations in the third $T^{2}$, they do not produce any fixed tori by themselves, but rather identify the fixed tori of $g_{\theta}$ and $g_{\omega}$ in pairs. The fixed tori of $g_{\theta}$ and $g_{\omega}$ are in one-to-one correspondence to the space group elements,

$$
\begin{aligned}
g_{r}=\left(\theta, n_{i} e_{i}\right) & \text { for } \quad n_{1}=n_{2}=0 \text { and } n_{3}, n_{4}, n_{6}=0,1, \\
g_{r^{\prime}}^{\prime}=\left(\omega, \frac{1}{2} e_{5}+n_{i} e_{i}\right) & \text { for } \quad n_{3}=n_{4}=0 \text { and } n_{1}, n_{2}, n_{6}^{\prime}=0,1 .
\end{aligned}
$$

We will often refer to the positions $\left(z_{1}, \frac{1}{2} n_{3}+\frac{i}{2} n_{4}, \frac{i}{2} n_{6}\right)$ and $\left(\frac{1}{2} n_{1}+\frac{i}{2} n_{2}, z_{2}, \frac{1}{4}+\frac{i}{2} n_{6}^{\prime}\right)$ of the fixed tori of $g_{\theta}$ and $g_{\omega}$ using multi-indices $r=\left(n_{3}, n_{4}, n_{6}\right)$ and $r^{\prime}=\left(n_{1}, n_{2}, n_{6}^{\prime}\right)$, respectively. As illustrated in figure 2 the fixed tori $r$ and $r^{\prime}$ lie parallel to each other in the third $T^{2}$. To emphasize this important fact we use the primes on $r^{\prime}$ and $n_{6}^{\prime}$ to signal that the 8 fixed points $r^{\prime}$ are shifted by $\frac{1}{4} e_{5}=\left(0,0, \frac{1}{4}\right)$ in the third torus w.r.t. the fixed points $r$.

When compactifying the heterotic string on this orbifold, massless strings are attached to these fixed tori as for the DW(0-1) orbifold above. However, since in this case the fixed tori do not intersect, there are no projections acting locally on the six-dimensional $\mathcal{N}=1$ theory. (Or more technically, even though $\theta$ and $\omega$ commute,the space group elements $g_{\theta}$ and $g_{\omega}$ do not, since they are defined to act on $\mathbb{R}^{6}$ not on $T^{6}$, see (2.12). Hence, the projection condition (2.10) is not implemented for these elements.) Nevertheless, from a four-dimensional point-of-view supersymmetry is broken to $\mathcal{N}=1$ : There are two sixdimensional theories living on the fixed tori of the $g_{\theta}$ and $g_{\omega}$ sectors which realize different six-dimensional $\mathcal{N}=1$ supersymmetries. Hence, in the effective four-dimensional theory only $\mathcal{N}=1$ remains. As this provides an example of non-local supersymmetry breaking, the six-dimensional hypermultiplets just branch into two chiral multiplets and consequently the resulting four-dimensional theory is necessarily non-chiral. Concretely, for the orbifold standard embedding we find for this orbifold $3+2 \cdot 8=19$ chiral 27-plets and $3+2 \cdot 8=19$ chiral $\overline{\mathbf{2 7}}$-plets of $\mathrm{E}_{6}$, i.e. the Hodge numbers are $(19,19)$. In fact, because supersymmetry is broken non-locally, any DW(0-2) orbifold is non-chiral, independently of the choice of shifts and Wilson lines.

Because of this, it would seem that this type of orbifold can never be relevant for fourdimensional model building. One of the main messages of this paper is that one should 
not discard such orbifolds for phenomenology just yet. In fact, as shown in section 5 it is possible to construct an explicit six generation SU(5) GUT model on the resolution of this orbifold. To break the GUT to the MSSM and to reduce the number of generations by a factor two one can use a (true field-theoretical) Wilson line $W_{\text {free }}$ associated to a free involution $\mathbb{Z}_{2 \text {,free }}$ of the geometry. In terms of the complex coordinates we take this involution to act as

$$
\left(z_{1}, z_{2}, z_{3}\right) \rightarrow\left(z_{1}+\frac{i}{2}, z_{2}+\frac{i}{2}, z_{3}+\frac{i}{2}\right)
$$

When this involution has been modded out the resulting geometry corresponds to the DW(1-3) orbifold with Hodge numbers $(11,11)$ in the classification [29]. In order that this is a symmetry of the full model, the discrete Wilson lines get severely restricted, i.e. $W_{2} \cong W_{4} \cong W_{6}$ and $W_{\text {free }} \cong \frac{1}{2} W_{2}$.

\section{Schoen manifold}

The Schoen manifold $X$ was first introduced in [15]; here we follow the description of this manifold given e.g. in [16-18, 37]. The Schoen manifold is defined as the fiber product

$$
X=B \times_{\mathbb{P}^{1}} B^{\prime},
$$

of two (four-dimensional) rational elliptic surfaces $B$ and $B^{\prime}$. Hence, the manifold $X$ is naturally equipped with two projections $\pi^{\prime}: X \rightarrow B$ and $\pi: X \rightarrow B^{\prime}$ that project on either factor of the fiber product. Such a rational elliptic surface $B$ is defined as a twotorus fibration $\beta: B \rightarrow \mathbb{P}^{1}$ over the base $\mathbb{P}^{1}$. In terms of the fibrations $\beta$ and $\beta^{\prime}$ the fiber product (3.1) is written as

$$
X:=\left\{\left(p, p^{\prime}\right) \in B \times B^{\prime} \mid \beta(p)=\beta^{\prime}\left(p^{\prime}\right)\right\} .
$$

A two-torus can be described as an elliptic curve, i.e. via the Weierstrass mapping as the solution to an homogeneous cubic polynomial constraint $f(x)=0$ in the homogeneous coordinates $x=\left(x_{0}, x_{1}, x_{2}\right) \in \mathbb{P}^{2}$. The complex structure of the torus is encoded in the constraint $f(x)=0$ : A homogeneous cubic polynomial is characterized by $3+6+1=10$ complex parameters, of which eight can be removed by complexified SU(3) rotations of $x$ and the overall complex scale is irrelevant. Because of the fibration the complex structure in general varies over the base $\mathbb{P}^{1}$. Therefore, the rational elliptic surface $B$ can be given by

$$
B=\mathbb{P}^{2}\left[\begin{array}{l}
3 \\
\mathbb{P}^{1}
\end{array}\right]: \quad B=\left\{p=(x, t) \in\left(\mathbb{P}^{2}, \mathbb{P}^{1}\right) \mid t_{0} f_{0}(x)-t_{1} f_{1}(x)=0\right\}
$$

The surface $B$ can thus be considered as the blow-up of $\mathbb{P}^{2}$ at (generically) $3 \cdot 3=9$ points $x$ where both cubic polynomials vanish simultaneously $f_{0}(x)=f_{1}(x)=0$. Since at each of these points an exceptional cycle $\mathbb{P}^{1}$ is inserted, the cohomology group $H^{2}(B, \mathbb{Z})=\mathbb{Z}^{10}$ is spanned by the hyper plane class $\ell$ of $\mathbb{P}^{2}$ and the exceptional classes $e_{\rho}(\rho=1, \ldots, 9)$ with intersection numbers $\ell^{2}=-e_{\rho}^{2}=1$. This surface has $c_{1}(B)=3 \ell-\sum_{\rho} e_{\rho}$ and Euler number $\chi(B)=c_{2}(B)=\chi\left(\mathbb{P}^{2}\right)+9 \cdot \chi\left(\mathbb{P}^{1}\right)=12$. 
As the description of $B^{\prime}$ is similar, the manifold $X$ can be written as a complete intersection Calabi-Yau

$$
X=\mathbb{P}^{2}\left[\begin{array}{ll}
3 & 0 \\
0 & 3 \\
1 & 1
\end{array}\right] \quad \mathbb{P}^{1} \quad\left\{\begin{array}{c}
t_{0} f_{0}(x)-t_{1} f_{1}(x)=0 \\
t_{0} f_{0}^{\prime}\left(x^{\prime}\right)-t_{1} f_{1}^{\prime}\left(x^{\prime}\right)=0
\end{array}\right.
$$

Clearly, the Calabi-Yau condition is satisfied as the horizontal sums of the degrees of the homogeneous polynomials are one higher than the dimension of the respective projective spaces. In this representation the number of complex structure deformations is readily counted: There are 4 cubic polynomials, $f_{0}(x), f_{1}(x), f_{0}^{\prime}\left(x^{\prime}\right), f_{1}^{\prime}\left(x^{\prime}\right)$, each containing 10 complex parameters. By redefinitions of $x, x^{\prime}$ and $t$ one can remove $2 \cdot 8+3$ of them and two overall complex scales are irrelevant, hence $h_{12}=4 \cdot 10-2 \cdot 8-3-2=19$. On both $B$ and $B^{\prime}$ there are $10(1,1)$-forms corresponding to the classes $\ell, e_{\rho}$ and $\ell^{\prime}, e_{\rho}^{\prime}$, respectively. However, since $\beta(p)=\beta^{\prime}\left(p^{\prime}\right)$ there is one linear relation among them. Consequently, the number of Kähler deformation equals $h_{11}=2 \cdot 10-1=19$. In summary the Hodge numbers of $X$ are $(19,19)$.

\subsection{Singular Schoen manifold: the DW(0-2) orbifold}

Putting the discussion above and the information obtained in subsection 2.3 together suggests that the $T^{6} / \mathbb{Z}_{2} \times \mathbb{Z}_{2, \text { rototrans }}$ orbifold can be considered as a specific singular limit of the Schoen manifold, since their Hodge numbers agree. Indeed, by the following considerations this can be confirmed [29]: On the covering six-torus coordinates $z$ the projects $\pi^{\prime}$ and $\pi$ act as $\pi^{\prime}(z)=\left(z_{1}, z_{3}\right)$ and $\pi(z)=\left(z_{2}, z_{3}\right)$, hence the rational elliptic surfaces $B$ and $B^{\prime}$ are given in this singular limit as

$$
\begin{aligned}
& B=\left\{\left(z_{1}, z_{3}\right) \in T_{1}^{2} \times T_{3}^{2} \mid\left(z_{1}, z_{3}\right) \sim\left(z_{1},-z_{3}\right) \sim\left(-z_{1}, \frac{1}{2}-z_{3}\right) \sim\left(-z_{1}, z_{3}+\frac{1}{2}\right)\right\} \\
& B^{\prime}=\left\{\left(z_{2}^{\prime}, z_{3}^{\prime}\right) \in T_{2}^{2} \times T_{3}^{2} \mid\left(z_{2}^{\prime}, z_{3}^{\prime}\right) \sim\left(z_{2}^{\prime}, \frac{1}{2}-z_{3}^{\prime}\right) \sim\left(-z_{2}^{\prime},-z_{3}^{\prime}\right) \sim\left(-z_{2}^{\prime}, z_{3}^{\prime}+\frac{1}{2}\right)\right\} .
\end{aligned}
$$

These spaces are isomorphic: By applying a change of coordinates $\left(z_{2}^{\prime}, z_{3}^{\prime}\right)=\left(z_{1}, z_{3}+\frac{1}{4}\right)$ the identifications in $B$ become identical to those in $B^{\prime}$. The $\mathbb{P}^{1}$ in the fiber product, which has the topology of a two-sphere, becomes a rectangular pillow

$$
\mathbb{P}^{1}=\left\{\left(z_{3}\right) \in T_{3}^{2} \mid\left(z_{3}\right) \sim\left(-z_{3}\right) \sim\left(\frac{1}{2}-z_{3}\right)\right\} .
$$

\subsection{Schoen manifold as the resolution of the DW(0-2) orbifold}

As has been explained e.g. in $[13,38,39]$ one can construct the smooth resolutions of compact orbifolds in a systematic fashion. In particular, it is possible to determine a convenient basis of divisors and the intersection numbers of the resolution of the orbifold 


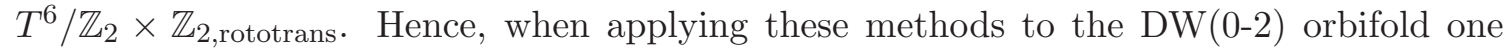
obtains a smooth Calabi-Yau space which constitutes a different realization of the Schoen manifold. As we will use this description of the Schoen manifold in the remainder of this paper, we describe this procedure in some detail below.

Intersection ring of a basis of divisors. The inherited divisors $R_{a}(a=1,2,3)$ correspond to the torus divisors $\left\{z_{a}=c_{a}\right\}$ with $c_{a}$ some generic complex numbers that are made compatible with the orbifold action:

$$
\begin{aligned}
& R_{1}:=\left\{z_{1}=c_{1}\right\} \cup\left\{z_{1}=-c_{1}\right\}, \quad R_{2}:=\left\{z_{2}=c_{2}\right\} \cup\left\{z_{2}=-c_{2}\right\}, \\
& R_{3}:=\left\{z_{3}=c_{3}\right\} \cup\left\{z_{3}=-c_{3}\right\} \cup\left\{z_{3}=\frac{1}{2}+c_{3}\right\} \cup\left\{z_{1}=\frac{1}{2}-c_{1}\right\},
\end{aligned}
$$

Next we define the ordinary divisors associated to the local coordinates defined near the fixed points of the orbifold

$$
\begin{aligned}
D_{1, n_{1} n_{2}} & :=\left\{z_{1}=\frac{1}{2} n_{1}+\frac{i}{2} n_{2}\right\}, \\
D_{2, n_{3} n_{4}} & :=\left\{z_{2}=\frac{1}{2} n_{3}+\frac{i}{2} n_{4}\right\}, \\
D_{3, n_{6}} & :=\left\{z_{3}=\frac{i}{2} n_{6}\right\} \cup\left\{z_{3}=\frac{1}{2}+\frac{i}{2} n_{6}\right\}, \\
D_{3, n_{6}^{\prime}}^{\prime} & :=\left\{z_{3}=\frac{1}{4}+\frac{i}{2} n_{6}^{\prime}\right\} \cup\left\{z_{3}=\frac{3}{4}+\frac{i}{2} n_{6}^{\prime}\right\} .
\end{aligned}
$$

Finally, the exceptional divisors, denoted by $E_{r^{\prime}}^{\prime}$ and $E_{r}$, arise when we resolve the $\mathbb{Z}_{2}$ singularities using e.g. toric geometry techniques. Since the fixed tori are identified in pairs in the DW(0-2) orbifold, we can represent them in terms of the exceptional divisors in the fundamental domain of the original $T^{6}$ (before modding out this identification):

$$
E_{r}=E_{n_{3} n_{4} n_{6}}=E_{n_{3} n_{4} 0 n_{6}} \cup E_{n_{3} n_{4} 1 n_{6}}, \quad E_{r^{\prime}}^{\prime}=E_{n_{1} n_{2} n_{6}^{\prime}}^{\prime}=E_{n_{1} n_{2} 0 n_{6}^{\prime}} \cup E_{n_{1} n_{2} 1 n_{6}^{\prime}} .
$$

These $8+8$ exceptional divisors stem from the $8+8$ fixed tori displayed in the figure 2 .

Between these divisors the following linear equivalence relations hold:

$$
\begin{array}{ll}
2 D_{1, n_{1} n_{2}}=R_{1}-\sum_{n_{6}^{\prime}} E_{n_{1} n_{2} n_{6}^{\prime}}^{\prime}, & 2 D_{3, n_{6}^{\prime}}^{\prime}=R_{3}-\sum_{n_{1}, n_{2}} E_{n_{1} n_{2} n_{6}^{\prime}}^{\prime}, \\
2 D_{2, n_{3} n_{4}}=R_{2}-\sum_{n_{6}} E_{n_{3} n_{4} n_{6}}, & 2 D_{3, n_{6}}=R_{3}-\sum_{n_{3}, n_{4}} E_{n_{3} n_{3} n_{6}} .
\end{array}
$$

These linear equivalence relations show that a basis for the $H_{2}(X, \mathbb{R})$ are formed by the divisors $R$ and $E$. (In total we have $3+2 \cdot 8=19$ of them.) In this basis the Schoen manifold has the following non-vanishing self-intersections between these divisors:

$$
R_{1} R_{2} R_{3}=4, \quad R_{2}\left(E_{n_{1} n_{2} n_{6}^{\prime}}^{\prime}\right)^{2}=R_{1}\left(E_{n_{3} n_{4} n_{6}}\right)^{2}=-4 .
$$

Using the linear equivalence relations (3.10) (self-)intersections between any combination of $R$ 's, $D$ 's and E's are readily computed. 
Chern classes. The total Chern class $\mathrm{c}(T X)$ of the tangent bundle of the resolution space $X$ can be computed from the splitting principle as

$$
\mathrm{c}(T X)=\prod(1+D) \prod(1+E) \prod(1-R)^{2},
$$

where the products are taken over all divisors of the appropriate types. By expanding this out, we can determine the first and second Chern classes of $X$. Using the linear equivalence relations (3.10) we find that $\mathrm{c}_{1}(T X)=0$, confirming that $X$ defines a Calabi-Yau space. For the second Chern class we obtain

$$
\mathrm{c}_{2}(T X)=-\frac{3}{4}\left(\sum_{r^{\prime}}\left(E_{r^{\prime}}^{\prime}\right)^{2}+\sum_{r}\left(E_{r}\right)^{2}\right)+\ldots
$$

The dots ... refer to further terms that appear in this expansion in principle, but which never contribute when integrated over any four-cycle using the intersection numbers given above.

By employing the adjunction formula, $\mathrm{c}_{2}(D)=D \mathrm{c}_{2}(X \mid D)$ one can determine the Euler number of the hyper surface associated to the divisor $D$. In particular, from

$$
\chi\left(R_{1}\right)=\mathrm{c}_{2}\left(R_{1}\right)=24, \quad \chi\left(R_{2}\right)=\mathrm{c}_{2}\left(R_{2}\right)=24, \quad \text { and } \quad \chi\left(R_{3}\right)=\mathrm{c}_{2}\left(R_{3}\right)=0,
$$

we infer that the divisors $R_{1}$ and $R_{2}$ are $K 3$ surfaces and $R_{3}$ is a four-torus. Finally, one may consider $D_{1,00}$ and $D_{2,00}$ as the divisor classes associated to the rational elliptic surfaces $B^{\prime}$ and $B$, respectively. Indeed, the Euler number of $D_{1,00}$ equals $\chi\left(D_{1,00}\right)=12$, and, since $D_{1,00} E_{r}^{2}=-2, D_{1,00}$ contains the same fixed points of $g_{\theta}$ as $B^{\prime}$, see (3.5). Hence, we may identify $B^{\prime}=D_{1,00}$ and similarly $B=D_{2,00}$.

\section{Line bundle models on the Schoen resolution}

In this section we consider Abelian gauge backgrounds on the Schoen geometry as described in the previous section. After that we determine the charged chiral spectrum in the presence of this background, showing in particular that even with line bundles it is possible to obtain chirality in four dimensions. Subsection 4.3 explains how the spectra of such line bundle backgrounds can be interpreted as heterotic orbifolds with appropriate blow-up modes switched on. The final subsection illustrates various aspects by giving an explicit line bundle model on the Schoen manifold.

\subsection{Abelian gauge flux backgrounds}

On the space $X$ we consider an Abelian gauge background $\mathcal{F}$ which is embedded in the Cartan subalgebra, spanned by the generators $H_{I}$, of the $\mathrm{E}_{8} \times \mathrm{E}_{8}{ }^{\prime}$ gauge group of the heterotic theory. In general this gauge flux is supported on both the exceptional and inherited divisors

$$
\frac{\mathcal{F}}{2 \pi}=\sum_{a} R_{a} H_{B_{a}}+\sum_{r} E_{r} H_{V_{r}}+\sum_{r^{\prime}} E_{r^{\prime}}^{\prime} H_{V_{r^{\prime}}^{\prime}} .
$$


The line bundle vectors $V_{r}, V_{r^{\prime}}^{\prime}$ and the magnetic fluxes $B_{a}$ on the tori are sixteendimensional component vectors that characterize the corresponding line bundle embedding in $\mathrm{E}_{8} \times \mathrm{E}_{8}{ }^{\prime}$. To shorten the notation we have written $H_{A}=A_{I} H_{I}$, where $A$ are referred to as bundle or flux vectors with 16 components, $A_{I}$.

In order that this gauge background is compatible with the freely acting $\mathbb{Z}_{2 \text {,free }}$ involution (2.14) of the orbifold, we need to require that

$$
V_{n_{3} n_{4} n_{6}}=V_{n_{3} n_{4}+1 n_{6}+1}, \quad V_{n_{1} n_{2} n_{6}^{\prime}}^{\prime}=V_{n_{1} n_{2}+1 n_{6}^{\prime}+1}^{\prime},
$$

Given that the indices take values $n_{i}=0,1$, the addition of indices is performed modulo 2 .

Flux quantization. For a gauge flux configuration (4.1) to be physically admissible it has to be integrally quantized, i.e.

$$
\int_{C} \frac{\mathcal{F}}{2 \pi} \in \mathbb{Z}
$$

for all curves $C$ that the manifold $X$ admits. This gives a stringent set of conditions on the bundle vectors

$$
\begin{aligned}
& 2 V_{n_{1} n_{2} n_{6}^{\prime}}^{\prime} \cong 2 V_{n_{3} n_{4} n_{6}} \cong 0, 2 B_{1} \cong 2 B_{2} \cong 2 B_{3} \cong 0, \\
& \sum_{n_{1}, n_{2}} V_{n_{1} n_{2} n_{6}^{\prime}}^{\prime}+B_{1} \cong \sum_{n_{3}, n_{4}} V_{n_{3} n_{4} n_{6}}+B_{2} \cong 0, \quad \sum_{n_{6}^{\prime}} V_{n_{1} n_{2} n_{6}^{\prime}}^{\prime}+\sum_{n_{6}} V_{n_{3} n_{4} n_{6}}+B_{3} \cong 0 .
\end{aligned}
$$

The first two conditions of (4.4a) are obtained by integrating over the curves $D_{2, n_{3} n_{4}} E_{n_{1} n_{2} n_{6}^{\prime}}^{\prime}$ and $D_{1, n_{1} n_{2}} E_{n_{3} n_{4} n_{6}}$, respectively. The first two sum conditions in (4.4b) result from integrating over $D_{2, n_{3} n_{4}} D_{3, n_{6}^{\prime}}^{\prime}$ and $D_{1, n_{1} n_{2}} D_{3, n_{6}}$, respectively. The last sum relation in (4.4b) is found by integrating over $D_{1, n_{1} n_{2}} D_{2, n_{3} n_{4}}$. When we combine these sum equations with the first two conditions of (4.4a), the latter three of (4.4a) are inferred. These equations are quite tricky to be solved in general. However, there are two (related) ansätze that simplify the problem considerably:

First of all, one may assume that the various bundle vectors are either equal or opposite, e.g.

$$
V_{n_{3} n_{4} n_{6}}=\sum_{s=0,1}(-)^{s\left(n_{4}+n_{6}\right)} V_{s n_{3}}, \quad V_{n_{1} n_{2} n_{6}^{\prime}}^{\prime}=\sum_{s^{\prime}=0,1}(-)^{s^{\prime}\left(n_{2}+n_{6}^{\prime}\right)} V_{s^{\prime} n_{1}}^{\prime} .
$$

This particular choice is compatible with the requirement (4.2) that the gauge fluxes admit the $\mathbb{Z}_{2 \text {,free }}$ action of equation (2.14). In this case, the sums of $V_{r}$ and $V_{r^{\prime}}^{\prime}$ in the conditions (4.4b) form lattice vectors. Consequently, the magnetic fluxes of the tori $B_{a}$ have to be lattice vectors themselves.

Secondly, taking inspiration from the expansion (2.4) of the local shift vectors $V_{g}$ in the orbifold construction, one may write the local bundle vectors as

$$
\begin{aligned}
& V_{n_{3} n_{4} n_{6}}=V_{\theta}+n_{3} W_{3}+n_{4} W_{4}+n_{6} W_{6}+L_{n_{3} n_{4} n_{6}}, \\
& V_{n_{1} n_{2} n_{6}^{\prime}}^{\prime}=V_{\omega}+n_{1} W_{1}+n_{2} W_{2}+n_{6}^{\prime} W_{6}+L_{n_{1} n_{2} n_{6}^{\prime}}^{\prime},
\end{aligned}
$$

where $2 V_{\theta} \cong 2 V_{\omega} \cong 2 W_{i} \cong 0$ and $L_{n_{3} n_{4} n_{6}} \cong L_{n_{1} n_{2} n_{6}^{\prime}}^{\prime} \cong 0$. Then, again, the sum conditions (4.4b) imply that the magnetic fluxes $B_{a}$ are lattice vectors. 
Bianchi identities. The central consistency requirements for smooth compactifications are the integrated Bianchi identities. In this work we ignore the possibility of five-branes, therefore the integrated Bianchi identities have to vanish for all divisors $D$, i.e.

$$
\int_{D}\left(\operatorname{tr} \mathcal{F}^{2}-\operatorname{tr} \mathcal{R}^{2}\right)=0
$$

Using the expression for the second Chern class (3.13) of $X$, we find in the present case that these conditions amount to

$$
\begin{aligned}
& B_{1} \cdot V_{r}=0, \\
& B_{2} \cdot V_{r^{\prime}}^{\prime}=0, \\
& B_{1} \cdot B_{2}=0 \text {, } \\
& \sum_{r}\left(V_{r}\right)^{2}=12+2 B_{2} \cdot B_{3}, \\
& \sum_{r^{\prime}}\left(V_{r^{\prime}}^{\prime}\right)^{2}=12+2 B_{1} \cdot B_{3},
\end{aligned}
$$

by integrating over $E_{r}, E_{r^{\prime}}, R_{3}$, and $R_{1}, R_{2}$, respectively.

The equations in (4.8a) show that the gauge fluxes $B_{1}$ and $B_{2}$ have to be perpendicular, and, that the gauge flux $B_{1}$ on $R_{1}$ is perpendicular to all the line bundle vectors $V_{r}$. Similarly, the gauge flux $B_{2}$ is perpendicular to all vectors $V_{r^{\prime}}^{\prime}$. However, there are no conditions on the inner products $B_{1} \cdot V_{r^{\prime}}^{\prime}$ and $B_{2} \cdot V_{r}$. The Bianchi identities on the second line, (4.8b), are reminiscent of the Bianchi identity on a single $\mathbb{C}^{2} / \mathbb{Z}_{2}$ resolution, i.e. $V^{2}=$ $3 / 2$ (see e.g. refs. [40,41]). For example, when $B_{2}$ or $B_{3}$ vanish and all eight $V_{r}$ are equal, this condition is reproduced identically. The magnetized tori thus lead to modifications of the standard local Bianchi identity of the local $\mathbb{C}^{2} / \mathbb{Z}_{2}$ resolution.

DUY equations and the blow-down limit. Using that the volume of a divisor $D$ is defined as $\operatorname{Vol}(D)=\int_{D} J^{2} / 2$, the tree-level DUY equation $\int J^{2} \mathcal{F}=0$ can be represented as

$$
\sum_{a} \operatorname{Vol}\left(R_{a}\right) B_{a}+\sum_{r} \operatorname{Vol}\left(E_{r}\right) V_{r}+\sum_{r^{\prime}} \operatorname{Vol}\left(E_{r^{\prime}}^{\prime}\right) V_{r^{\prime}}^{\prime}=0 .
$$

These conditions can be very restrictive. They give an equal number of relations between the volumes as the number of linear independent vectors the $B_{a}, V_{r}$ and $V_{r^{\prime}}^{\prime}$ can be decomposed in. However, one such relation may force many volumes to zero simultaneously, because these volumes are of course assumed to be non-negative.

As we describe the Schoen manifold as a resolution of the $T^{6} / \mathbb{Z}_{2} \times \mathbb{Z}_{2 \text {,rototrans, we }}$ would like to determine the requirements under which a gauge flux configuration allows for a regular blow-down limit in which the underlying six torus $T^{6}$ has a finite volume. Hence, we search for solutions which allow for a full blow-down to the singular orbifold, i.e. with $\operatorname{Vol}\left(E_{r}\right)=\operatorname{Vol}\left(E_{r^{\prime}}^{\prime}\right)=0$ and $\operatorname{Vol}\left(R_{a}\right)>0$. In this case, the DUY equations simplify to

$$
\operatorname{Vol}\left(R_{1}\right) B_{1}+\operatorname{Vol}\left(R_{2}\right) B_{2}+\operatorname{Vol}\left(R_{3}\right) B_{3}=0 .
$$

It follows that unless the $B_{a}$ are linearly dependent or all zero, at least some of the volumes $\operatorname{Vol}\left(R_{a}\right)$ are forced to vanish. In particular, if only one $B_{a}$ is non-zero, then the corresponding volume has to be zero in the blow-down limit, and hence a regular blow-down limit does not exist. Even when $B_{3}$ is a linear combination of $B_{1}$ and $B_{2}$ but one of the coefficients is positive, two volumes are forced to zero. Hence, only if $B_{3}$ is a linear combination 
with negative coefficients of $B_{1}$ and $B_{2}$, a regular blow-down limit exists. Hence, possibly the simplest way to realize this has $B_{3}=-B_{1}-B_{2}$. We can cast this in the form of two equations

$$
B_{1}^{2} \operatorname{Vol}\left(R_{1}\right)+B_{1} \cdot B_{3} \operatorname{Vol}\left(R_{3}\right)=0, \quad B_{2}^{2} \operatorname{Vol}\left(R_{2}\right)+B_{2} \cdot B_{3} \operatorname{Vol}\left(R_{3}\right)=0,
$$

since $B_{1}$ and $B_{2}$ are perpendicular, see (4.8a). Since we know from (4.4a) that $2 B_{a} \cong 0$, we see that the ratios of the volumes of the inherited divisors, $\operatorname{Vol}\left(R_{a}\right) / \operatorname{Vol}\left(R_{3}\right), a=1,2$, are fractional.

To allow for a full blow-up we need in addition that the fluxes located at the exceptional divisors to be chosen such that the volumes of all of them can be taken to be positive at the same time. To ensure this it is again convenient to choose that the corresponding bundle vectors are alternating, e.g. like in (4.5).

It turns out that the combination of the flux quantization, DUY equations and the Bianchi identities is extremely restrictive, hence, to obtain semi-realistic models, one is often forced to give up the requirement of a regular blow-down limit. (In addition, the oneloop correction to the DUY equation [26] can force some volumes to be non-vanishing.) When this limit does not exist, an orbifold interpretation of the model is not ruled out: Often it is possible to shrink quite a number of exceptional cycles to zero, while keeping the volumes $\operatorname{Vol}\left(R_{a}\right)>0$. Hence, locally near those shrunken cycles a non-compact orbifold analysis is still possible.

\subsection{Spectra computation}

To determine the physical consequence of models build on such orbifold resolutions we need to be able to determine the spectrum of massless states. A convenient way of computing the spectrum on an orbifold resolution is provided by the multiplicity operator introduced e.g. in [40-42]. Using these methods we can determine both the spectra in four dimensions as well as on six-dimensional hyper surfaces.

Four-dimensional spectrum. The spectrum in four dimensions is of key interest in phenomenological studies. It can be determined by letting the operator

$$
N_{4 D}(X)=\int_{X}\left\{\frac{1}{6}\left(\frac{\mathcal{F}}{2 \pi}\right)^{3}+\frac{1}{12} c_{2}(T X) \frac{\mathcal{F}}{2 \pi}\right\}
$$

act on the states contained in the ten-dimensional gaugino. This operator is normalized such that it counts the number of chiral superfields. Using the intersection numbers determined above this is computed straightforwardly:

$$
N_{4 D}(X)=2\left(1-\sum_{r} H_{V_{r}}^{2}\right) H_{B_{1}}+2\left(1-\sum_{r^{\prime}} H_{V_{r^{\prime}}^{\prime}}^{2}\right) H_{B_{2}}+4 H_{B_{1}} H_{B_{2}} H_{B_{3}} .
$$

The multiplicities of the chiral multiplets in four dimensions are then determined by evaluating this operator on the roots of $\mathrm{E}_{8} \times \mathrm{E}_{8}{ }^{\prime}$.

There is some tension between solving the Bianchi identities and chirality, because of the orthogonality relations, (4.8a), among the fluxes $B_{a}, V_{r}$ and $V_{r^{\prime}}^{\prime}$. However, say 
$B_{1} \cdot V_{r}=0$, does not imply that on all $\mathrm{E}_{8} \times \mathrm{E}_{8}^{\prime}$ roots $H_{B_{1}} H_{V_{r}}^{2}$ vanishes. As we show by some examples discussed in the section 5 , it is indeed possible to obtain a chiral spectrum in four dimensions.

Six-dimensional spectra on divisors. In addition, we can define the multiplicity operator $N_{6 D}(D)$ in six dimensions for any divisor $D \subset X$. Positive values of $N_{6 D}$ count the number of half-hyper multiplets, while negative values count (two times) the number of vector multiplets. (In six dimensions the fermions of hyper and vector multiplets have opposite chirality.) As integrals over the whole space $X$ they read

$$
N_{6 D}(D)=\int_{X} D\left\{\frac{1}{2}\left(\frac{\mathcal{F}}{2 \pi}\right)^{2}+\frac{1}{12} c_{2}(D)\right\},
$$

where $\mathrm{c}_{2}(D)$ are given in (3.14). Given that the divisors $R_{1}, R_{2}$ may be interpreted as $K 3$ surfaces and $R_{3}$ as a four-torus, the spectra on these divisors are probably the most interesting. Using the intersection numbers we readily compute this explicitly for $D=R_{a}$ :

$$
\begin{aligned}
& N_{6 D}\left(R_{1}\right)=2\left(1-\sum_{r} H_{V_{r}}^{2}\right)+4 H_{B_{2}} H_{B_{3}}, \\
& N_{6 D}\left(R_{2}\right)=2\left(1-\sum_{r^{\prime}} H_{V_{r^{\prime}}^{\prime}}^{2}\right)+4 H_{B_{1}} H_{B_{3}}, \\
& N_{6 D}\left(R_{3}\right)=4 H_{B_{1}} H_{B_{2}} .
\end{aligned}
$$

Relation between the six- and four-dimensional spectra. As explained in sub-

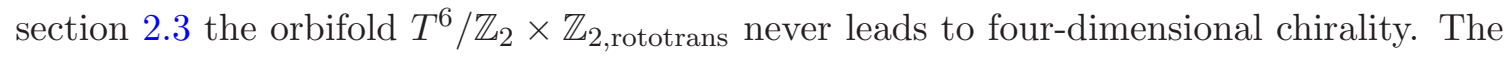
reason is basically that such models only contain hypermultiplets in six dimension, which simply branch to vector-like combinations of chiral multiplets in four dimensions. In the smooth case we have found a way to bypass this no-go. The key here are the magnetic fluxes $B_{a}$ on the divisors $R_{a}$ that correspond to the tori of the orbifold in the blow-down limit. Indeed, if we set all $B_{a}=0$, then (4.13) simply says that $N_{4 D}=0$ : no chirality. Hence, precisely by allowing for magnetized divisors $R_{a}$ we can avoid this no-go and obtain chirality.

To see that this effect is expected from field theory, let us consider the case in which the flux $B_{2}$ has been switched off. The four-dimensional multiplicity operator (4.13) then leads to a relation between the six-dimensional spectrum on $R_{1}$ given by (4.15a) and the spectrum in four dimensions:

$$
N_{4 D}(X)=H_{B_{1}} N_{6 D}\left(R_{1}\right)
$$

This equation can be interpreted as follows: When we compactify on a $K 3 \times T^{2}$, then we can consider first the six-dimensional theory that results from the compactification on $K 3$. This effective six-dimensional model is subsequently reduced on a two-torus. It is wellknown that if there is no magnetic flux, this second step results in a vector-like spectrum in four dimensions. However, if there is a magnetic flux $B$ present, a chiral spectrum arises: only the chiral fermionic states of charge $q$ for which $B q>0$ survive, and their multiplicity 
is given by $B q$ provided that the smallest charge in the spectrum is unity [34, 36]. It is intriguing to notice that (4.16) says exactly this: $N_{6 D}\left(R_{1}\right)$ determines the spectrum in a six-dimensional world. The operator $H_{B_{1}}$ gives the charge $B_{1} \cdot w$ of the six-dimensional state associated with the $\mathrm{E}_{8} \times \mathrm{E}_{8}{ }^{\prime}$ root $w$ under the magnetic flux $B_{1}$.

When all three fluxes are switched on, the relation between the four- and sixdimensional spectra apparently reads

$$
N_{4 D}(X)=H_{B_{1}} N_{6 D}\left(R_{1}\right)+H_{B_{2}} N_{6 D}\left(R_{2}\right)-H_{B_{3}} N_{6 D}\left(R_{3}\right) .
$$

This follows directly by identifying the six-dimensional multiplicity operators (4.15) in the four-dimensional expression (4.13). The final term corrects for over counting of states charged under $B_{1}, B_{2}$ and $B_{3}$ simultaneously.

\subsection{Interpretation as blow-up of DW(0-2) orbifold models}

So far, we have analyzed the Schoen geometry as a smooth Calabi-Yau and described line bundle backgrounds on it. The fact that the Schoen manifold is the resolution of the DW(02 ) orbifold, as discussed in section 3 , has essentially been irrelevant in our investigation. Now, we would like to describe how a given line bundle model can be understood as a heterotic DW(0-2) orbifold model with a certain number of blow-up modes attaining vacuum expectation values (VEVs). We first recall how this analysis can be done in general using a $\mathcal{N}=1$ language in four dimensions following [10,13, 42]. After that we conclude this subsection by describing this procedure in a six-dimensional supersymmetric formulation which is more appropriate since the DW(0-2) orbifold model has $\mathcal{N}=1$ supersymmetric sectors in six dimensions.

Four dimensional $\mathcal{N}=1$ language. In heterotic orbifolds a fixed point gets blown up if a twisted chiral superfield $\Phi_{\mathrm{bm}}^{(r)}$, localized at that fixed point, acquires a non-vanishing VEV: $\left\langle\Phi_{\mathrm{bm}}^{(r)}\right\rangle \neq 0$. The value of this VEV determines the volume of the exceptional cycle $E_{r}$ that appears in this resolution process. As we recalled in section 2.1 any twisted state is characterized by a shifted left-moving momentum $P_{\text {sh }}$. In refs. $[10,13,43]$ it was realized that, as long as this twisted state does not involve any oscillator excitations, its shifted momentum $P_{\text {sh }}$ precisely determines the local line bundle vector $V_{r}$ associated to the exceptional divisor $E_{r}$. Some special cases might occur: Sometimes it happens that a bundle vector corresponds to a blow-up mode that has been projected out by the orbifold action in the four-dimensional theory. It is also possible that the bundle vector is associated to a massive state in the orbifold spectrum.

The spectrum of the orbifold model and the one of the blow-up theory are generically not identical, but closely related: First of all the VEVs of the twisted states $\left\langle\Phi_{\mathrm{bm}}^{(r)}\right\rangle$ lead to some gauge symmetry breaking. Furthermore, the blow-up modes are not present in the blow-up spectrum as charged states, but rather as (complexified) axions $b_{r}$. The relation between the blow-up mode and the axion reads

$$
\Phi_{\mathrm{bm}}^{(r)}=e^{b_{r}}\left\langle\Phi_{\mathrm{bm}}^{(r)}\right\rangle .
$$


In the smooth description this axion generically gives a mass to the gauge field of the broken $U(1)$ via the Stueckelberg mechanism. In the blow-up picture the $U(1)$ is broken just by a standard Higgs mechanism.

As a consequence of this gauge symmetry breaking the representations of matter fields get branched. But still, this is not enough to match the orbifold and resolution spectra $[10,13]$ : One needs to preform field redefinitions of the other twisted matter states $\Phi_{\text {orb }}$ involving the corresponding blow-up modes to obtain an exact agreement of the spectra, i.e.

$$
\Phi_{\text {orb }}=e^{ \pm b_{r}} \Phi_{\text {res }} .
$$

The signs \pm have to be chosen appropriately to ensure that the weights of $\Phi_{\text {res }}$ are $\mathrm{E}_{8} \times \mathrm{E}_{8}{ }^{\prime}$ roots, while those of $\Phi_{\text {orb }}$ belong to the shifted weight lattice defined in (2.9).

Blow-ups in six dimensions. Before we describe the blow-up procedure in a sixdimensional language, we first briefly recall some properties of $\mathcal{N}=1$ theories in six dimensions. There are three basic irreducible representation of $\mathcal{N}=1$ supersymmetry relevant for our discussion: i) A vector multiplet $\mathcal{V}=(\mathrm{V}, \Phi)$ contains a vector superfield $\mathrm{V}$ and a chiral superfield $\Phi$ from the $4 \mathrm{D} \mathcal{N}=1$ perspective. ii) A hypermultiplet contains two independent chiral superfields $\mathcal{H}=\left(\Phi, \Phi^{c}\right)$ that live in charge-conjugate representations. This means that the gauge properties of the hypermultiplet is uniquely specified by the representation and U(1) charges of either chiral component. iii) Finally, a half-hyper multiplet is a hypermultiplet with a certain reality condition imposed. Therefore, it has only half of the number of independent components as a normal hypermultiplet. In other words, using the four-dimensional $\mathcal{N}=1$ terminology, a half-hyper is a chiral superfield in a real or pseudo-real representation.

Now, if a twisted hypermultiplet plays the role of a blow-up mode in order to resolve a fixed torus, then only one of its chiral superfield components actually takes a VEV, while the other component only gets redefined:

$$
\mathcal{H}_{\mathrm{bm}}^{(r)}=e^{b_{r}}\left(\left\langle\Phi_{\mathrm{bm}}^{(r)}\right\rangle, \Phi_{\mathrm{bm}, \mathrm{res}}^{c(r)}\right) .
$$

Because the chiral superfield components of a hypermultiplet carry opposite U(1) charges, they have to be redefined with opposite powers of the blow-up mode:

$$
\mathcal{H}_{\mathrm{orb}}=\left(\Phi_{\mathrm{orb}}, \Phi_{\mathrm{orb}}^{c}\right)=\left(e^{ \pm b_{r}} \Phi_{\mathrm{res}}, e^{\mp b_{r}} \Phi_{\text {res }}^{c}\right),
$$

for appropriate choice of sign \pm . After these field redefinitions the chiral superfields in the blow-up mode hypermultiplet do not seem to fall into proper $\mathcal{N}=1$ representations anymore. However, this does not signify that the blow-up breaks six-dimensional supersymmetry: The remaining chiral superfield components will be completely neutral, and therefore form half-hypermultiplets by themselves.

\subsection{Sample model: an eight generation GUT}

We conclude this section with a concrete example of a line bundle model which is constructed on the Schoen geometry to illustrate many aspects of the general description 
developed in this and the preceding sections. Consider the following particular line bundle model on the resolution of our $T^{6} / \mathbb{Z}_{2} \times \mathbb{Z}_{2 \text {,rototrans: }}$

$$
B_{3}=-B_{1}, \quad B_{2}=0, \quad V_{n_{3} n_{4} n_{6}}=(-)^{n_{4}+n_{6}} V_{0}, \quad V_{n_{1} n_{2} n_{6}^{\prime}}^{\prime}(-)^{n_{2}+n_{6}^{\prime}} V_{n_{1}}^{\prime},
$$

with

$$
\begin{array}{rlrl}
B_{1} & =\left(1,1,1,-1,0,0^{3}\right)\left(0^{8}\right), & V_{0}^{\prime}=\left(0,0,0, \frac{1}{2}, \frac{1}{2}, 0^{3}\right)\left(0^{8}\right), \\
V_{0}=\left(0,0, \frac{1}{2}, \frac{1}{2}, 0,0^{3}\right)\left(1,0^{7}\right), & V_{1}^{\prime}=\left(\frac{1}{2},-\frac{1}{2}, 0,0,0,0^{3}\right)\left(0^{8}\right) .
\end{array}
$$

This model exhibits the following properties:

The bundle vectors satisfy all the requirements specified in section 4.1: The quantization conditions (4.4) are fulfilled, because the alternating signs in the $V_{r}$ and $V_{r^{\prime}}^{\prime}$ are in accordance with (4.5) and the vectors $B_{a}$ are lattice vectors.

They also satisfy all Bianchi identities (4.8): $B_{1}$ is perpendicular to all vectors $V_{r}$. Since all vectors $V_{r}$ square to $3 / 2$ and $B_{2}=0$ the first condition in (4.8b) is satisfied. The second condition in $(4.8 \mathrm{~b})$ is satisfied as well, since both sides are equal:

$$
\sum_{r^{\prime}} V_{r^{\prime}}^{\prime 2}=8 \cdot \frac{1}{2}=4, \quad 12+2 B_{1} B_{3}=12-2 \cdot 4=4
$$

Because $B_{3}=-B_{1}$ and $B_{2}=0$ a blow-down of this model is allowed by the DUY equations (4.9) while keeping the torus radii, set by the volumes of the divisors $R_{a}$, finite. In the blow-down limit, $\operatorname{Vol}\left(E_{r}\right)=\operatorname{Vol}\left(E_{r^{\prime}}^{\prime}\right)=0$, the volumes of $R_{1}$ and $R_{3}$ have to be equal, $\operatorname{Vol}\left(R_{3}\right)=\operatorname{Vol}\left(R_{1}\right)$. The alternating signs of $V_{r}$ and $V_{r^{\prime}}^{\prime}$ ensure that the DUY equations also allow for a finite blow-up of all exceptional cycles.

The gauge group that is left unbroken by this Abelian gauge configuration is

$$
\mathrm{SU}(5) \times \mathrm{SO}(14)^{\prime} \times \mathrm{U}(1)^{5}
$$

from the first and second $\mathrm{E}_{8}$ group factor. Since for this choice of bundle vectors $B_{2}=0$ and all $V_{r}$ are equal up to a sign, the $4 \mathrm{D}$ multiplicity operator (4.13) reduces to

$$
N_{4 D}=2 H_{B_{1}}\left(1-8 H_{V_{0}}^{2}\right) .
$$

The resulting spectrum,

$$
8(\mathbf{1 0}, \mathbf{1})+12(\overline{\mathbf{5}}, \mathbf{1})+4(\mathbf{5}, \mathbf{1})+24(\mathbf{1}, \mathbf{1}),
$$

is chiral w.r.t. to the five $\mathrm{U}(1)$ charges (which we omitted for notational simplicity). (W.r.t. the hidden gauge group at most a purely vector-like spectrum arises, which is invisible for the multiplicity operator.) Hence, the model might be considered as an eight generations SU(5) GUT toy-model with four Higgs pairs. 


\section{A line bundle MSSM on the Schoen manifold}

We present an MSSM-like model with three generations as a line bundle model on the reso-

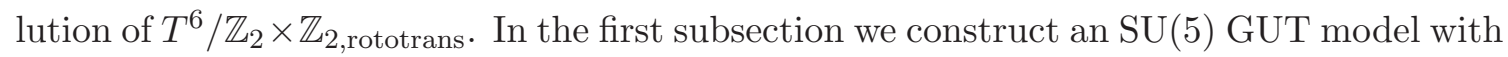
six generations on the Schoen manifold using line bundles. In subsection 5.2 we identify a Wilson line that can be associated with a freely acting involution, which both reduces the number of generations to three and breaks the gauge group to the standard model group. In the next subsection we show that a $K 3$ subspace of the Schoen manifold can be blown down to a four-dimensional orbifold $T^{4} / \mathbb{Z}_{2}$ on which the model can be quantized using standard CFT techniques. In subsection 5.4 we use this to give an alternative description of the line bundle MSSM on the Schoen manifold in terms of a blow-up of this orbifold with a magnetized torus.

\subsection{Six GUT generations on the Schoen resolution}

We define a line bundle model on the Schoen manifold with the flux vectors

$$
B_{1}=\left(3,-3,0^{6}\right)\left(3,3,0^{6}\right) \text { and } \quad B_{2}=B_{3}=0,
$$

on the ordinary divisors $R_{a}$,

$$
\begin{aligned}
& V_{(0,0,0)}=V_{(0,1,0)}=-V_{(0,0,1)}=-V_{(0,1,1)}=\left(\frac{1}{4}^{8}\right)\left(0,0,0, \frac{1}{2}, 0,-\frac{1}{2},-\frac{1}{2},-\frac{1}{2}\right), \\
& V_{(1,0,0)}=V_{(1,1,0)}=-V_{(1,0,1)}=-V_{(1,1,1)}=\left(0, \frac{1}{2}, \frac{1}{2}, 0^{5}\right)\left(0, \frac{1}{2}, 0,0,0,-\frac{1}{2},-\frac{1}{2},-\frac{1}{2}\right),
\end{aligned}
$$

on the exceptional divisors $E_{r}$, and finally,

$$
\begin{gathered}
V_{(0,0,0)}^{\prime}=-V_{(0,1,1)}^{\prime}=\left(0,-\frac{1}{2},-\frac{1}{2}, 0^{5}\right)\left(\frac{1}{2}, \frac{1}{2}, \frac{1}{2}, 0,-\frac{1}{2}, 0,0,0\right), \\
V_{(0,1,0)}^{\prime}=-V_{(0,0,1)}^{\prime}=\left(0,-\frac{1}{2},-\frac{1}{2}, 0^{5}\right)\left(\frac{1}{2}, \frac{1}{2},-\frac{1}{2}, 0, \frac{1}{2}, 0,0,0\right), \\
V_{(1,0,0)}^{\prime}=V_{(1,1,0)}^{\prime}=\left(0,1,0,0^{5}\right)\left(-\frac{1}{2},-\frac{1}{2}, 0,0,0,0,0,0\right), \\
V_{(1,1,1)}^{\prime}=V_{(1,0,1)}^{\prime}=\left(-1,0^{7}\right)\left(-\frac{1}{2},-\frac{1}{2}, 0^{6}\right),
\end{gathered}
$$

on the exceptional divisors $E_{r^{\prime}}^{\prime}$.

This choice of bundle vectors fulfills the quantization conditions (4.4) and the DUY equations (4.9) for appropriately chosen volumes. All bundle vectors $V_{r}$ and $V_{r^{\prime}}^{\prime}$ have $V_{r}^{2}=V_{r^{\prime}}^{\prime 2}=3 / 2$. This is consistent with the Bianchi identities $(4.8 \mathrm{~b})$, which reduce to

$$
\sum_{r}\left(V_{r}\right)^{2}=\sum_{r^{\prime}}\left(V_{r^{\prime}}^{\prime}\right)^{2}=12
$$




\begin{tabular}{|c|c|cccccccc|}
\hline Superfield & Representation & \multicolumn{1}{|c|}{ U(1) charges } \\
multiplicity & SU(5) $\times$ SU $(5)^{\prime}$ & $q_{0}$ & $q_{1}$ & $q_{2}$ & $q_{3}$ & $q_{4}$ & $q_{5}$ & $q_{6}$ & $q_{7}$ \\
\hline 6 & $(\overline{\mathbf{1 0}}, \mathbf{1})$ & 0 & 0 & 0 & 0 & 1 & 0 & -3 & 0 \\
6 & $(\mathbf{5}, \mathbf{1})$ & 0 & 0 & 0 & 0 & 0 & 0 & -6 & 0 \\
6 & $(\overline{\mathbf{5}}, \mathbf{1})$ & 1 & 0 & 1 & 0 & -1 & 0 & 1 & 0 \\
6 & $(\mathbf{5}, \mathbf{1})$ & 1 & 0 & 1 & 0 & 0 & 0 & 4 & 0 \\
24 & $(\mathbf{1}, \mathbf{1})$ & 2 & 0 & 0 & 0 & 0 & 0 & 0 & 0 \\
6 & $(\mathbf{1}, \mathbf{1})$ & -1 & 0 & -1 & 0 & -1 & 0 & 5 & 0 \\
6 & $(\mathbf{1}, \mathbf{1})$ & 1 & 0 & -3 & 0 & 0 & 0 & 0 & 0 \\
6 & $(\mathbf{1}, \mathbf{1})$ & 0 & 0 & 0 & 0 & 2 & 0 & 0 & 0 \\
\hline 6 & $(\mathbf{1}, \overline{\mathbf{1 0}})$ & 0 & 0 & 0 & 2 & 0 & 0 & 0 & -6 \\
24 & $(\mathbf{1}, \mathbf{5})$ & 0 & 1 & 0 & 3 & 0 & 0 & 0 & -2 \\
6 & $(\mathbf{1}, \overline{\mathbf{5}})$ & 0 & 0 & 0 & -2 & 0 & 0 & 0 & -8 \\
6 & $(\mathbf{1}, \overline{\mathbf{5}})$ & 0 & 0 & 0 & 0 & 0 & 1 & 0 & 7 \\
6 & $(\mathbf{1}, \overline{\mathbf{5}})$ & 0 & 0 & 0 & 0 & 0 & -1 & 0 & 7 \\
42 & $(\mathbf{1}, \mathbf{1})$ & 0 & 0 & 0 & 4 & 0 & 1 & 0 & -5 \\
42 & $(\mathbf{1}, \mathbf{1})$ & 0 & 0 & 0 & 4 & 0 & -1 & 0 & -5 \\
24 & $(\mathbf{1}, \mathbf{1})$ & 0 & 1 & 0 & -3 & 0 & 1 & 0 & -5 \\
24 & $(\mathbf{1}, \mathbf{1})$ & 0 & 1 & 0 & -3 & 0 & -1 & 0 & -5 \\
6 & $(\mathbf{1}, \mathbf{1})$ & 0 & 2 & 0 & 0 & 0 & 0 & 0 & 0 \\
\hline
\end{tabular}

Table 1. This line bundle model on the Schoen manifold has six generations of SU(5) in both, the observable and the hidden, sectors. States in the first block are charged under the observable $\mathrm{E}_{8}$; states in the second block are charged under the hidden group.

since there are no corrections resulting from magnetic fluxes $B_{a}$ as only $B_{1} \neq 0$. The unbroken gauge group in this gauge configuration reads

$$
\mathrm{SU}(5) \times \mathrm{SU}(5)^{\prime} \times \mathrm{U}(1)^{8} .
$$

The four-dimensional multiplicity operator (4.13) is computed straightforwardly and the resulting chiral spectrum is given in table 1 . In this table we have distinguished the various states, in particular the singlets, by their eight $\mathrm{U}(1)$ charges $\left(q_{0}, \ldots, q_{7}\right)$. Notice that, curiously, this model has six generations in both, the observable and the hidden, SU(5).

\subsection{Freely acting $\mathbb{Z}_{2}$ and MSSM with three generations}

One can define a freely acting involution $\mathbb{Z}_{2, \text { free }}$ as in equation (2.14) that reduces the number of generations by a factor $1 / 2$. In addition, the freely acting involution can be embedded as a Wilson line that breaks $\mathrm{SU}(5)$ to $\mathrm{SU}(3) \times \mathrm{SU}(2) \times \mathrm{U}(1)_{Y}$. We take this 
Wilson line,

$$
W_{\text {free }}=\left(0^{3}, 1,1,1,-\frac{3}{2},-\frac{3}{2}\right)\left(0^{8}\right),
$$

to point in the standard hypercharge direction of $\mathrm{SU}(5)$. This choice of $W_{\text {free }}$ fixes the first $\mathrm{SU}(5)$ to define the observable sector and leads to an MSSM-like model with three generations.

Contrary to the situation in field theory, there are further requirements on this Wilson line in string theory $[12,13]$ : It has to satisfy $2 W_{\text {free }} \cong W_{2} \cong W_{4} \cong W_{6} \cong 0$ and it has to respect the modular invariance conditions

$$
2 W_{\text {free }}^{2} \equiv W_{\text {free }} \cdot W_{i} \equiv 0
$$

These additional conditions were derived in context of orbifold constructions where $\mathbb{Z}_{2 \text {,free }}$ is part of the space group.

\subsection{Singular limits of the Schoen GUT with line bundles}

Full blow down limit. Taking the magnetic flux $B_{1}$ to vanish for a moment, we can consider the full blow-down limit of the GUT model with six generations. It has an exact

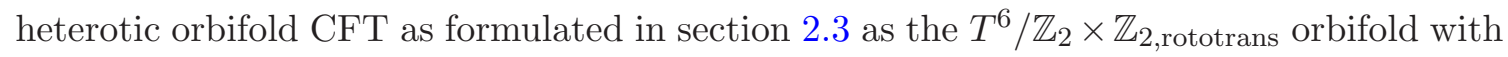
a definite choice of gauge shifts, $V_{\theta}, V_{\omega}$, and discrete Wilson lines, $W_{i}$. As dictated by the flux vectors (5.2) and (5.3) they are given by

$$
\begin{aligned}
V_{\theta} & =\left(\frac{1}{4}^{8}\right)\left(\frac{1}{2},-\frac{1}{2}, \frac{1}{2}, 0, \frac{1}{2}, 0^{3}\right), \\
V_{\omega} & =\left(0,-\frac{1}{2},-\frac{1}{2}, 0^{5}\right)\left(-\frac{1}{2},-\frac{1}{2},-\frac{1}{2}, 0, \frac{1}{2}, 0^{3}\right), \\
W_{1} & =\left(0, \frac{1}{2},-\frac{1}{2}, 1,1,0^{3}\right)\left(0,1, \frac{1}{2}, 1,-\frac{1}{2}, 0^{3}\right), \\
W_{3} & =\left(-\frac{1}{4}, \frac{1}{4}, \frac{1}{4},-\frac{1^{5}}{4}\right)\left(0, \frac{1}{2}, 0, \frac{1}{2},-1,0^{3}\right),
\end{aligned}
$$

and the other Wilson lines vanish. This choice fulfills the conditions of modular invariance (2.7). As discussed in section 2.3, the spectrum of this orbifold can be computed using orbifold CFT techniques but is necessarily non-chiral as long as no magnetic fluxes $B_{a}$ have been reintroduced.

The $T^{4} / \mathbb{Z}_{2}$ orbifold inside the Schoen manifold. Since the Schoen model defined in this section has only a single magnetic flux, $B_{1}$, switched on, see (5.1), the DUY equations (4.9) imply that in a full blow-down the volume of $R_{1}$ has to vanish as well. However, we can exploit that there also exists a partial blow-down in which all $\operatorname{Vol}\left(E_{r}\right) \rightarrow 0$ while the volumes of all inherited divisors $R_{a}$ and of at least some other exceptional divisors $E_{r^{\prime}}^{\prime}$ stay finite. Therefore, this partial blow-down leads to an intermediate $T^{4} / \mathbb{Z}_{2}$ orbifold with torus coordinates $\left(z_{2}, z_{3}\right)$ on which the $\mathbb{Z}_{2}$ action acts via the twist $v_{\theta}$ given in (2.3) (c.f. [11]). 
For this intermediate $T^{4} / \mathbb{Z}_{2}$ orbifold an exact heterotic CFT description exists. Taking its gauge embedding as given by $V_{\theta}$ and $W_{3}$ from equation (5.8), its low energy limit results in a model with $\mathcal{N}=1$ supersymmetry in six dimensions with gauge group

$$
\mathrm{E}_{6} \times \mathrm{SU}(8)^{\prime} \times \mathrm{U}(1)^{3} .
$$

The spectrum of hypermultiplets including $U(1)$ charges of this intermediate sixdimensional orbifold theory is computed using [7] and listed in the first column of table 2.

A simple, yet non-trivial crosscheck of this spectrum is that it is free of irreducible gravitational anomalies, e.g. that the sum condition \#(hyper) $-\#$ (vector) $=244$ holds. Indeed, using table 2 it is straightforward to count the number of vector- and hypermultiplets:

$$
\begin{aligned}
& \#(\text { vector })=78+63+3 \cdot 1=144, \\
& \#(\text { hyper })=2 \cdot\left(27+\frac{1}{2} \cdot 70+2\right)+16 \cdot 2 \cdot 8+4=388
\end{aligned}
$$

where the factor $\frac{1}{2}$ accounts for the fact that the $(\mathbf{1}, \mathbf{7 0})_{(0,0,0)}$ is a half-hyper. The last 4 additional hypers correspond to untwisted moduli which are not displayed in table 2.

\subsection{Schoen line bundle MSSM as a blown up orbifold}

The MSSM-like model of subsection 5.1 can now be reproduced as a blow-up of the $T^{4} / \mathbb{Z}_{2}$ orbifold discussed in the subsection above equipped with a magnetic flux on the torus to generate four-dimensional chirality. In short, this procedure reads:

1. Blow-up the $T^{4} / \mathbb{Z}_{2}$ orbifold to a smooth $K 3$ manifold by giving VEVs to 16 blow-up modes and use field redefinitions to obtain the spectrum on $K 3$.

2. Turn on the additional fluxes on the divisors $E_{r^{\prime}}^{\prime}$, decompose gauge group and branch the representations accordingly.

3. Generate four-dimensional chirality by switching on the magnetic flux $B_{1}$ as well.

In this process the magnetic flux $B_{1}$ does not lead to breaking of four-dimensional supersymmetry since the contribution from the fluxes on $E_{r^{\prime}}^{\prime}$ cancels the one from $B_{1}$ in the DUY equations (4.9). In the following we describe this procedure in detail:

Blowing up the intermediate $T^{4} / \mathbb{Z}_{2}$ orbifold. The intermediate $T^{4} / \mathbb{Z}_{2}$ orbifold gets blown up to a $K 3$ surface by assigning VEVs to the blow-up modes, i.e. to 16 twisted states localized at the 16 singularities of the $T^{4} / \mathbb{Z}_{2}$ orbifold. At each singularity (labeled by the multi-index $\left.r=\left(n_{3}, n_{4}, n_{5}, n_{6}\right)\right)$ a blow-up mode, $\Phi_{\mathrm{bm}}^{(r)}$ contained in a twisted hypermultiplet, is chosen such that its shifted left-moving momentum $P_{\mathrm{sh}}^{(r)}$ agrees with the flux vector $V_{r}$ localized on the divisor $E_{r}$ :

$$
P_{\mathrm{sh}}^{(r)}=V_{r} \quad \text { for all } \quad r=\left(n_{3}, n_{4}, n_{5}, n_{6}\right)
$$


All these blow-up modes are chosen to be in eight-dimensional representations of the hidden $\mathrm{SU}(8)^{\prime}$ gauge group of the $T^{6} / \mathbb{Z}_{2}$ orbifold, so that, consequently, the gauge group gets broken to

$$
\mathrm{E}_{6} \times \mathrm{SU}(7)^{\prime} \times \mathrm{U}(1)^{4}
$$

As explained in subsection 4.3, when the blow-up mode $\Phi_{\mathrm{bm}}^{(r)}$ in a given twisted sector attains a VEV, field redefinitions, (4.20) and (4.21), have to be performed on the other states in the same twisted sector in order to ensure that all fields in the blow-up are characterized by $\mathrm{E}_{8} \times \mathrm{E}_{8}{ }^{\prime}$ roots. The appropriate field redefinitions required by this blow-up procedure are listed in the second column of table 2 . As the phases of the orbifold blowup modes have been reinterpreted as axions, the remaining hypermultiplet components do not seem to form proper six-dimensional $\mathcal{N}=1$ hypermultiplets. However, as can be verified from this table, these chiral superfield are neutral and can thus be interpreted as half-hypermultiplets.

Additional fluxes on the exceptional divisors $\boldsymbol{E}_{r^{\prime}}^{\prime}$. Up to now, we have turned on fluxes only on the exceptional divisors $E_{r}$, which correspond to the blown-up fixed points of the $T^{4} / \mathbb{Z}_{2}$ orbifold. After the field redefinition to the blow-up field basis, the charges w.r.t. to the four $\mathrm{U}(1)$ factors are taken such that they correspond to the first four charges $\left(q_{0}, q_{1}, q_{2}, q_{3}\right)$ in table 1 . The U(1)'s associated to the charges $\left(q_{0}, q_{1}, q_{2}\right)$ already exist at the intermediate $T^{4} / \mathbb{Z}_{2}$ orbifold, the fourth $\mathrm{U}(1)$ arises by symmetry breaking of the hidden $\mathrm{SU}(8)^{\prime}$ in the blow-up procedure.

Turning on additional fluxes (5.3) on $E_{r^{\prime}}^{\prime}$ induces a further gauge symmetry breaking to

$$
\mathrm{SU}(5) \times \mathrm{SU}(5)^{\prime} \times \mathrm{U}(1)^{8}
$$

Since, these fluxes are located at resolved fixed points of the other orbifold twist $v_{\omega}$, they respect a different six-dimensional supersymmetry. This means that by switching on these fluxes the model becomes $\mathcal{N}=1$ in four dimensions. However, because the divisors $E_{r^{\prime}}^{\prime}$ do not intersect with $E_{r}$ (i.e. the fixed tori of the $g_{\theta^{-}}$and $g_{\omega^{-}}$-twisted sectors do not intersect) see figure 2, this does not enforce any chiral projection on the matter spectrum (in contrast to, say, the gravitino): The matter states on $K 3$ (i.e. the blow-up of the intermediate $\left.T^{4} / \mathbb{Z}_{2}\right)$ are simply decomposed into four-dimensional superfields and their representations are branched according to the symmetry breaking (5.13).

Generating four dimensional chirality. States from $E_{r}$ feel the flux on their "fixed torus", i.e. on $R_{1}$, so that the $B_{1}$ flux induces chirality in four dimensions. Whether a state is part of the charged chiral spectrum is decided by the operator

$$
\tilde{N}_{4 D}=2 H_{B_{1}}
$$

In general $[34,36]$, if $\tilde{N}_{4 D}$ is positive for a chiral superfield $\Phi_{r e s}$, then $\tilde{N}_{4 D}$ copies of $\Phi_{\text {res }}$ appear in the four-dimensional spectrum. While, if $\tilde{N}_{4 D}$ is negative $\Phi_{\text {res }}$ is completely projected out. Thus this relation (similarly to (4.16)) shows that four dimensional chirality only arises if the flux $B_{1}$ is switched on. 


\begin{tabular}{|c|c|c|c|}
\hline $\begin{array}{c}6 \mathrm{D} \mathcal{N}=1 \text { super } \\
\text { multiplet on } T^{4} / \mathbb{Z}_{2} \\
\left(\mathrm{E}_{6} \times \mathrm{SU}(8)^{\prime} \times \mathrm{U}(1)^{3}\right)\end{array}$ & $\begin{array}{l}\text { Blow-up induced redefinitions of } \\
\text { its chiral superfield component(s) } \\
\qquad\left(\mathrm{E}_{6} \times \mathrm{SU}(7)^{\prime} \times \mathrm{U}(1)^{4}\right)\end{array}$ & \begin{tabular}{|c|} 
Surviving $4 \mathrm{D}$ \\
chiral superfields \\
$\left(\mathrm{SU}(5) \times \mathrm{SU}(5)^{\prime} \times \mathrm{U}(1)^{8}\right)$
\end{tabular} & $\begin{array}{l}4 \mathrm{D} \text { multi- } \\
\text { plicity } \\
\tilde{N}_{4 D}\end{array}$ \\
\hline \multicolumn{4}{|c|}{ untwisted gauge sector } \\
\hline $\begin{array}{c}(\mathbf{7 8}, \mathbf{1})_{(0,0,0)} \\
\quad(\text { vector })\end{array}$ & $(\mathbf{7 8}, \mathbf{1})_{(0,0,0)}$ & $\begin{array}{c}(\overline{\mathbf{1 0}}, \mathbf{1})_{(0,0,0,0,1,0,-3,0)} \\
(\mathbf{5}, \mathbf{1})_{(0,0,0,0,0,0,-6,0)} \\
(\mathbf{1}, \mathbf{1})_{(0,0,0,0,2,0,0,0)}\end{array}$ & $\begin{array}{l}6 \\
6 \\
6\end{array}$ \\
\hline \multirow[t]{4}{*}{$\begin{array}{c}\mathbf{1}, \mathbf{6 3})_{(0,0,0)} \\
(\text { vector })\end{array}$} & $(\mathbf{1}, \mathbf{4 8})_{(0,0,0,0)}$ & $\begin{array}{l}(\mathbf{1}, \overline{\mathbf{5}})_{(0,0,0,0,0,1,0,7)} \\
(\mathbf{1}, \overline{\mathbf{5}})_{(0,0,0,0,0,-1,0,7)}\end{array}$ & $\begin{array}{l}6 \\
6\end{array}$ \\
\hline & $(\mathbf{1}, \overline{\mathbf{7}})_{(0,0,0,4)}$ & - & - \\
\hline & $(\mathbf{1}, \mathbf{7})_{(0,0,0,-4)}$ & $\begin{array}{l}(\mathbf{1}, \mathbf{1})_{(0,0,0,-4,0,1,0,5)} \\
(\mathbf{1}, \mathbf{1})_{(0,0,0,-4,0,-1,0,5)}\end{array}$ & $\begin{array}{l}6 \\
6\end{array}$ \\
\hline & $(\mathbf{1}, \mathbf{1})_{(0,0,0,0)}$ & - & - \\
\hline \multicolumn{4}{|c|}{ untwisted matter sectors: $U_{a}, a=2,3$} \\
\hline \multirow{2}{*}{$\begin{array}{l}(\mathbf{2 7}, \mathbf{1})_{(-1,0,-1)} \\
\quad \text { (hyper) }\end{array}$} & $(\mathbf{2 7}, \mathbf{1})_{(-1,0,-1,0)}$ & $(\mathbf{1}, \mathbf{1})_{(-1,0,-1,0,-1,0,5,0)}$ & 6 \\
\hline & $(\overline{\mathbf{2 7}}, \mathbf{1})_{(1,0,1,0)}$ & $\begin{array}{l}(\mathbf{5}, \mathbf{1})_{(1,0,1,0,0,0,4,0)} \\
(\overline{\mathbf{5}}, \mathbf{1})_{(1,0,1,0,-1,0,1,0)}\end{array}$ & $\begin{array}{l}6 \\
6\end{array}$ \\
\hline \multirow{2}{*}{$\begin{array}{l}(\mathbf{1}, \mathbf{7 0})_{(0,0,0)} \\
\text { (half-hyper) }\end{array}$} & $(\mathbf{1}, \overline{\mathbf{3 5}})_{(0,0,0,-2)}$ & $(\mathbf{1}, \overline{\mathbf{5}})_{(0,0,0,-2,0,0,0,-8)}$ & 6 \\
\hline & $(\mathbf{1}, \mathbf{3 5})_{(0,0,0,2)}$ & $(\mathbf{1}, \overline{\mathbf{1 0}})_{(0,0,0,2,0,0,0,-6)}$ & 6 \\
\hline \multirow{2}{*}{$\begin{array}{c}(\mathbf{1}, \mathbf{1})_{(1,0,-3)} \\
\text { (hyper) }\end{array}$} & $(\mathbf{1}, \mathbf{1})_{(1,0,-3,0)}$ & $(\mathbf{1}, \mathbf{1})_{(1,0,-3,0,0,0,0,0)}$ & 6 \\
\hline & $(\mathbf{1}, \mathbf{1})_{(-1,0,3,0)}$ & - & - \\
\hline \multirow{2}{*}{$\begin{array}{c}(\mathbf{1}, \mathbf{1})_{(0,2,0)} \\
\text { (hyper) }\end{array}$} & $(\mathbf{1}, \mathbf{1})_{(0,2,0,0)}$ & $(\mathbf{1}, \mathbf{1})_{(0,2,0,0,0,0,0,0)}$ & 6 \\
\hline & $(\mathbf{1}, \mathbf{1})_{(0,-2,0,0)}$ & - & - \\
\hline \multicolumn{4}{|c|}{ twisted matter sector at the fixed tori: $r=\left(0, n_{4}, n_{5}, 0\right), n_{4}, n_{5}=0,1$} \\
\hline \multirow{4}{*}{$\begin{array}{c}(\mathbf{1}, \mathbf{8}) \\
\left(-\frac{1}{2},-\frac{1}{2},-\frac{3}{2}\right) \\
\text { (hyper) }\end{array}$} & $(\mathbf{1}, \mathbf{1})_{\left(\frac{1}{2}, \frac{1}{2}, \frac{3}{2},-\frac{7}{2}\right)}=e^{+b_{r}}$ & blow-up mode & axion \\
\hline & $(\mathbf{1}, \mathbf{1})_{\left(-\frac{1}{2},-\frac{1}{2},-\frac{3}{2}, \frac{7}{2}\right)}=e^{+b_{r}}(\mathbf{1}, \mathbf{1})_{(0,0,0,0)}$ & - & - \\
\hline & $(\mathbf{1}, \mathbf{7})_{\left(-\frac{1}{2},-\frac{1}{2},-\frac{3}{2},-\frac{1}{2}\right)}=e^{+b_{r}}(\mathbf{1}, \mathbf{7})_{(0,0,0,-4)}$ & - & - \\
\hline & $(\mathbf{1}, \overline{\mathbf{7}})_{\left(\frac{1}{2}, \frac{1}{2}, \frac{3}{2}, \frac{1}{2}\right)}=e^{-b_{r}}(\mathbf{1}, \overline{\mathbf{7}})_{(0,0,0,4)}$ & $\begin{array}{l}(\mathbf{1}, \mathbf{1})_{(0,0,0,4,0,1,0,-5)} \\
(\mathbf{1}, \mathbf{1})_{(0,0,0,4,0,-1,0,-5)}\end{array}$ & 6 \\
\hline \multirow{4}{*}{$\begin{array}{l}(\mathbf{1}, \mathbf{8})\left(\frac{1}{2},-\frac{1}{2}, \frac{3}{2}\right) \\
\text { (hyper) }\end{array}$} & $(\mathbf{1}, \mathbf{1})_{\left(\frac{1}{2},-\frac{1}{2}, \frac{3}{2}, \frac{7}{2}\right)}=e^{+b_{r}}(\mathbf{1}, \mathbf{1})_{(1,0,3,0)}$ & - & - \\
\hline & $(\mathbf{1}, \mathbf{1})_{\left(-\frac{1}{2}, \frac{1}{2},-\frac{3}{2},-\frac{7}{2}\right)}=e^{-b_{r}}(\mathbf{1}, \mathbf{1})_{(-1,0,-3,0)}$ & - & - \\
\hline & $(\mathbf{1}, \overline{\mathbf{7}})_{\left(-\frac{1}{2}, \frac{1}{2},-\frac{3}{2}, \frac{1}{2}\right)}=e^{+b_{r}}(\mathbf{1}, \overline{\mathbf{7}})_{(0,1,0,-3)}$ & $\begin{array}{l}(\mathbf{1}, \mathbf{1})_{(0,1,0,-3,0,1,0,-5)} \\
(\mathbf{1}, \mathbf{1})_{(0,1,0,-3,0,-1,0,-5)}\end{array}$ & $\begin{array}{l}6 \\
6\end{array}$ \\
\hline & $(\mathbf{1}, \mathbf{7})_{\left(\frac{1}{2},-\frac{1}{2}, \frac{3}{2},-\frac{1}{2}\right)}=e^{-b_{r}}(\mathbf{1}, \mathbf{7})_{(0,-1,0,3)}$ & - & - \\
\hline
\end{tabular}

Table 2. (Continues...) 


\begin{tabular}{|c|c|c|c|}
\hline $\begin{array}{c}6 \mathrm{D} \mathcal{N}=1 \text { super } \\
\text { multiplet on } T^{4} / \mathbb{Z}_{2} \\
\left(\mathrm{E}_{6} \times \mathrm{SU}(8)^{\prime} \times \mathrm{U}(1)^{3}\right)\end{array}$ & $\begin{array}{l}\text { Blow-up induced redefinitions of } \\
\text { its chiral superfield component(s) } \\
\qquad\left(\mathrm{E}_{6} \times \mathrm{SU}(7)^{\prime} \times \mathrm{U}(1)^{4}\right)\end{array}$ & $\begin{array}{c}\text { Surviving } 4 \mathrm{D} \\
\text { chiral superfields } \\
\left(\mathrm{SU}(5) \times \mathrm{SU}(5)^{\prime} \times \mathrm{U}(1)^{8}\right)\end{array}$ & $\begin{array}{c}4 \mathrm{D} \text { multi- } \\
\text { plicity } \\
\tilde{N}_{4 D}\end{array}$ \\
\hline \multicolumn{4}{|c|}{ twisted matter sector at the fixed tori: $r=\left(0, n_{4}, n_{5}, 1\right), n_{4}, n_{5}=0,1$} \\
\hline \multirow{4}{*}{$\begin{array}{c}(\mathbf{1}, \mathbf{8})\left(-\frac{1}{2},-\frac{1}{2},-\frac{3}{2}\right) \\
\text { (hyper) }\end{array}$} & $(\mathbf{1}, \mathbf{1})_{\left(-\frac{1}{2},-\frac{1}{2},-\frac{3}{2}, \frac{7}{2}\right)}=e^{+b_{r}}$ & blow-up mode & axion \\
\hline & $(\mathbf{1}, \mathbf{1})_{\left(\frac{1}{2}, \frac{1}{2}, \frac{3}{2},-\frac{7}{2}\right)}=e^{+b_{r}}(\mathbf{1}, \mathbf{1})_{(0,0,0,0)}$ & - & - \\
\hline & $\left.\frac{3}{2}, \frac{1}{2}\right)=e^{+b_{r}}(\mathbf{1}, \overline{\mathbf{7}})_{(0,0,0,4)}$ & $\begin{array}{l}(\mathbf{1}, \mathbf{1})_{(0,0,0,4,0,1,0,-5)} \\
(\mathbf{1}, \mathbf{1})_{(0,0,0,4,0,-1,0,-5)}\end{array}$ & 6 \\
\hline & $(\mathbf{1}, \mathbf{7})_{\left(-\frac{1}{2},-\frac{1}{2},-\frac{3}{2},-\frac{1}{2}\right)}=e^{-b_{r}}(\mathbf{1}, \mathbf{7})_{(0,0,0,-4)}$ & - & - \\
\hline \multirow{4}{*}{$\begin{array}{l}(\mathbf{1}, \mathbf{8}) \\
\left(\frac{1}{2},-\frac{1}{2}, \frac{3}{2}\right) \\
\text { (hyper) }\end{array}$} & $(\mathbf{1}, \mathbf{1})_{\left(-\frac{1}{2}, \frac{1}{2},-\frac{3}{2},-\frac{7}{2}\right)}=e^{+b_{r}}(\mathbf{1}, \mathbf{1})_{(-1,0,-3,0)}$ & - & - \\
\hline & $(\mathbf{1}, \mathbf{1})_{\left(\frac{1}{2},-\frac{1}{2}, \frac{3}{2}, \frac{7}{2}\right)}=e^{-b_{r}}(\mathbf{1}, \mathbf{1})_{(1,0,3,0)}$ & - & - \\
\hline & $(\mathbf{1}, \mathbf{7})_{\left(\frac{1}{2},-\frac{1}{2}, \frac{3}{2},-\frac{1}{2}\right)}=e^{+b_{r}}(\mathbf{1}, \mathbf{7})_{(0,-1,0,3)}$ & - & - \\
\hline & $(\mathbf{1}, \overline{\mathbf{7}})_{\left(-\frac{1}{2}, \frac{1}{2},-\frac{3}{2}, \frac{1}{2}\right)}=e^{-b_{r}}(\mathbf{1}, \overline{\mathbf{7}})_{(0,1,0,-3)}$ & $\begin{array}{l}(\mathbf{1}, \mathbf{1})_{(0,1,0,-3,0,1,0,-5)} \\
(\mathbf{1}, \mathbf{1})_{(0,1,0,-3,0,-1,0,-5)}\end{array}$ & 6 \\
\hline \multicolumn{4}{|c|}{ twisted matter sector at the fixed tori: $r=\left(1, n_{4}, n_{5}, 0\right), n_{4}, n_{5}=0,1$} \\
\hline \multirow{4}{*}{$\begin{array}{c}(\mathbf{1}, \mathbf{8})\left(-1, \frac{1}{2}, 0\right) \\
\text { (hyper) }\end{array}$} & $(\mathbf{1}, \mathbf{1})_{\left(1,-\frac{1}{2}, 0,-\frac{7}{2}\right)}=e^{+b_{r}}$ & blow-up mode & axion \\
\hline & $(\mathbf{1}, \mathbf{1})_{\left(-1, \frac{1}{2}, 0, \frac{7}{2}\right)}=e^{+b_{r}}(\mathbf{1}, \mathbf{1})_{(0,0,0,0)}$ & - & - \\
\hline & $\left.{ }^{(\mathbf{1}, \mathbf{7}}\right)_{\left(-1, \frac{1}{2}, 0,-\frac{1}{2}\right)}=e^{+b_{r}}(\mathbf{1}, \mathbf{7})_{(0,0,0,-4)}$ & - & - \\
\hline & $(\mathbf{1}, \overline{\mathbf{7}})_{\left(1,-\frac{1}{2}, 0, \frac{1}{2}\right)}=e^{-b_{r}}(\mathbf{1}, \overline{\mathbf{7}})_{(0,0,0,4)}$ & $\begin{array}{l}(\mathbf{1}, \mathbf{1})_{(0,0,0,4,0,1,0,-5)} \\
(\mathbf{1}, \mathbf{1})_{(0,0,0,4,0,-1,0,-5)}\end{array}$ & 6 \\
\hline \multirow{4}{*}{$\begin{array}{c}(\mathbf{1}, \mathbf{8})\left(1, \frac{1}{2}, 0\right) \\
\text { (hyper) }\end{array}$} & $(\mathbf{1}, \mathbf{1})_{\left(1, \frac{1}{2}, 0, \frac{7}{2}\right)}=e^{+b_{r}}(\mathbf{1}, \mathbf{1})_{(2,0,0,0)}$ & $(\mathbf{1}, \mathbf{1})_{(2,0,0,0,0,0,0,0)}$ & 6 \\
\hline & $(\mathbf{1}, \mathbf{1})_{\left(-1,-\frac{1}{2}, 0,-\frac{7}{2}\right)}=e^{-b_{r}}(\mathbf{1}, \mathbf{1})_{(-2,0,0,0)}$ & - & - \\
\hline & $(\mathbf{1}, \overline{\mathbf{7}})_{\left(-1,-\frac{1}{2}, 0, \frac{1}{2}\right)}=e^{+b_{r}}(\mathbf{1}, \overline{\mathbf{7}})_{(0,-1,0,-3)}$ & - & - \\
\hline & $(\mathbf{1}, \mathbf{7})_{\left(1, \frac{1}{2}, 0,-\frac{1}{2}\right)}=e^{-b_{r}}(\mathbf{1}, \mathbf{7})_{(0,1,0,3)}$ & $(\mathbf{1}, \mathbf{5})_{(0,1,0,3,0,0,0,-2)}$ & 6 \\
\hline \multicolumn{4}{|c|}{ twisted matter sector at the fixed tori: $r=\left(1, n_{4}, n_{5}, 1\right), n_{4}, n_{5}=0,1$} \\
\hline \multirow{4}{*}{$\begin{array}{c}(\mathbf{1}, \mathbf{8})\left(-1, \frac{1}{2}, 0\right) \\
\text { (hyper) }\end{array}$} & $(\mathbf{1}, \mathbf{1})_{\left(-1, \frac{1}{2}, 0, \frac{7}{2}\right)}=e^{+b_{r}}$ & blow-up mode & axion \\
\hline & $(\mathbf{1}, \mathbf{1})_{\left(1,-\frac{1}{2}, 0,-\frac{7}{2}\right)}=e^{+b_{r}}(\mathbf{1}, \mathbf{1})_{(0,0,0,0)}$ & - & - \\
\hline & $(\mathbf{1}, \overline{\mathbf{7}})_{\left(1,-\frac{1}{2}, 0, \frac{1}{2}\right)}=e^{+b_{r}}(\mathbf{1}, \overline{\mathbf{7}})_{(0,0,0,4)}$ & $\begin{array}{l}(\mathbf{1}, \mathbf{1})_{(0,0,0,4,0,1,0,-5)} \\
(\mathbf{1}, \mathbf{1})_{(0,0,0,4,0,-1,0,-5)}\end{array}$ & 6 \\
\hline & $(\mathbf{1}, \mathbf{7})_{\left(-1, \frac{1}{2}, 0,-\frac{1}{2}\right)}=e^{-b_{r}}(\mathbf{1}, \mathbf{7})_{(0,0,0,-4)}$ & - & - \\
\hline
\end{tabular}

Table 2. (Continues...) 


\begin{tabular}{|c|c|c|c|}
\hline $\begin{array}{c}6 \mathrm{D} \mathcal{N}=1 \text { super } \\
\text { multiplet on } T^{4} / \mathbb{Z}_{2} \\
\left(\mathrm{E}_{6} \times \mathrm{SU}(8)^{\prime} \times \mathrm{U}(1)^{3}\right)\end{array}$ & $\begin{array}{l}\text { Blow-up induced redefinitions of } \\
\text { its chiral superfield component }(\mathrm{s}) \\
\qquad\left(\mathrm{E}_{6} \times \mathrm{SU}(7)^{\prime} \times \mathrm{U}(1)^{4}\right)\end{array}$ & $\begin{array}{c}\text { Surviving } 4 \mathrm{D} \\
\text { chiral superfields } \\
\left(\mathrm{SU}(5) \times \mathrm{SU}(5)^{\prime} \times \mathrm{U}(1)^{8}\right)\end{array}$ & $\begin{array}{l}4 \mathrm{D} \text { multi- } \\
\text { plicity } \\
\tilde{N}_{4 D}\end{array}$ \\
\hline \multirow{4}{*}{$\begin{array}{c}(\mathbf{1}, \mathbf{8})\left(1, \frac{1}{2}, 0\right) \\
\text { (hyper) }\end{array}$} & $(\mathbf{1}, \mathbf{1})_{\left(-1,-\frac{1}{2}, 0,-\frac{7}{2}\right)}=e^{+b_{r}}(\mathbf{1}, \mathbf{1})_{(-2,0,0,0)}$ & - & - \\
\hline & $(\mathbf{1}, \mathbf{1})_{\left(1, \frac{1}{2}, 0, \frac{7}{2}\right)}=e^{-b_{r}}(\mathbf{1}, \mathbf{1})_{(2,0,0,0)}$ & $(\mathbf{1}, \mathbf{1})_{(2,0,0,0,0,0,0,0)}$ & 6 \\
\hline & $(\mathbf{1}, \mathbf{7})_{\left(1, \frac{1}{2}, 0,-\frac{1}{2}\right)}=e^{+b_{r}}(\mathbf{1}, \mathbf{7})_{(0,1,0,3)}$ & $(\mathbf{1}, \mathbf{5})_{(0,1,0,3,0,0,0,-2)}$ & 6 \\
\hline & $(\mathbf{1}, \overline{\mathbf{7}})_{\left(-1,-\frac{1}{2}, 0, \frac{1}{2}\right)}=e^{-b_{r}}(\mathbf{1}, \overline{\mathbf{7}})_{(0,-1,0,-3)}$ & - & - \\
\hline
\end{tabular}

Table 2. The first column gives $6 \mathrm{D} \mathcal{N}=1$ multiplets on the $T^{4} / \mathbb{Z}_{2}$ orbifold with twist $g_{\theta}$ and gauge embedding $V_{\theta}$ and $W_{3}$ from equation (5.8). The second column indicates which state is the blow-up mode and gives the field redefinitions necessary to match the orbifold and blow-up states. In the third column we only indicate the states which are part of the four-dimensional chiral spectrum, i.e. those for which $\tilde{N}_{4 D}$, given in the last column, is positive.

Two important observations are in order: Chiral multiplets originating from the sixdimensional vector multiplets get an extra factor $(-1)$ in order to account for the different chiralities of vector and hypermultiplets in six dimensions. In addition, note that the effect of the Wilson line $W_{1}$ in the presence of a flux $B_{1}$ in the same torus can be seen in a field theoretical approach as a shift in the wave-functions. Hence, concerning the spectrum of massless modes it can be neglected.

As the 16 fixed points of $T^{4} / \mathbb{Z}_{2}$ are identified pairwise by the $\mathbb{Z}_{2}$ action of $g_{\omega}$, one has to restrict to twisted states with $n_{5}=0$. Furthermore, $g_{\omega}$ projects out all states from the untwisted sector $U_{2}$ as can be seen in the full orbifold model $T^{6} / \mathbb{Z}_{2} \times \mathbb{Z}_{2 \text {,rototrans. The }}$ result of the additional fluxes is listed in the third and fourth columns of table 2. The chiral part of the resulting spectrum agrees with the spectrum of the smooth model listed in table 1.

\section{Towards an CFT description of orbifolds with magnetized tori}

In this section we propose modifications to the standard CFT construction of heterotic orbifolds in the presence of magnetized tori. To facilitate this discussion we first recall a few standard facts of heterotic orbifolds, i.e. orbifolds without any magnetic flux supported on the two-tori, $B_{a}=0$.

\subsection{Standard modular invariance conditions}

The conditions of modular invariance are compatible with the local Bianchi identities in the absence of $B_{a}$-fluxes in the following sense: If we choose space group elements $g=h=g_{r}$ or $g_{r^{\prime}}$, as defined in equation (2.13) we see from (2.6) that the associated local shifts $V_{g_{r}}$ and $V_{g_{r^{\prime}}}$ fulfill

$$
V_{g_{r}}^{2} \equiv \frac{3}{2}, \quad V_{g_{r^{\prime}}}^{2} \equiv \frac{3}{2},
$$

where $r, r^{\prime}$ label the $8+8$ fixed points of the twisted sectors of $\theta$ and $\omega$, respectively. On the other hand, in the smooth picture if we assume gauge fluxes $V_{r} \cong V_{g_{r}}$ and $V_{r^{\prime}}^{\prime} \cong V_{g_{r^{\prime}}}$ 
of length-square $3 / 2$ at all $8+8$ resolved fixed points $r$ and $r^{\prime}$, respectively, then modular invariance corresponds (modulo integers) to $1 / 8$ th of the Bianchi identities (4.8b) with $B_{a}=0$.

\subsection{Heterotic description of the Schoen orbifold with magnetized tori}

Inspired by the logic put forward in [44] we propose how the modular invariance conditions (2.6) are modified in the presence of magnetically charged tori, $B_{a} \neq 0$. Since the magnetic fluxes are constant over the tori, it is natural to assume that at a given fixed point they only contribute as one over the number of fixed points, i.e. 1/8. As can be inferred from the local Bianchi identities (4.8b) the magnetic fluxes, $B_{a}$, contribute to the energy (12 is replaced by $12+2 B_{a} \cdot B_{3}, a=1,2$ ). Hence, we propose that the local modular invariance conditions (6.1) are modified to

$$
V_{g_{r}}^{2} \equiv \frac{3}{2}+\frac{1}{4} B_{2} \cdot B_{3}, \quad V_{g_{r^{\prime}}}^{2} \equiv \frac{3}{2}+\frac{1}{4} B_{1} \cdot B_{3},
$$

In order to satisfy the quantization conditions (4.4) and the DUY equations in blowdown (4.10) it is convenient to expand $B_{3}$ as a linear combination of $B_{1}$ and $B_{2}$ with negative coefficients. According to equation (6.2) this reduces the lengths of the local shifts $V_{g_{r}}$ and $V_{g_{r^{\prime}}}$. For example, using e.g. $B_{3}=-B_{1}-B_{2}$ yields $V_{g_{r}}^{2} \equiv \frac{3}{2}-\frac{1}{4} B_{2}^{2}$.

However, as we have seen in blow-up not only the consistency conditions, i.e. the Bianchi conditions, get modified in the presence of $B_{a}$-fluxes, but also the spectra. Therefore, one could imagine that the mass shell condition (2.8) on orbifolds is modified as well when $B_{a} \neq 0$. In analogy to the proposal in [44], we expect that the left-moving mass is modified to

$$
M_{L}^{2}=\frac{1}{2}\left(P+V_{g_{r}}\right)^{2}+\widetilde{N}-\frac{3}{4}-\frac{1}{8} B_{2} \cdot B_{3}, \quad M_{L}^{2}=\frac{1}{2}\left(P+V_{g_{r^{\prime}}}\right)^{2}+\widetilde{N}-\frac{3}{4}-\frac{1}{8} B_{1} \cdot B_{3} .
$$

If we follow the interpretation of the local line bundle vectors as the shifted momenta (2.9) of twisted states that generate the blow-up at $r$ or $r^{\prime}$ these equations will contribute new twisted states as blow-up modes, which where not part of the $B_{a}=0$ orbifold spectrum.

When one considers the standard heterotic orbifold, (massless) states, that survive the level matching condition, are subject to the orbifold projection conditions (2.10). Modifications of these projections are, as far as we are aware, not discussed in the literature. Moreover, since it is unknown how the heterotic string is quantized in the presence of magnetized tori, there is also not an obvious computation that would determine the appropriate corrections. However, as usual we expect that at least self-projections, i.e. taking $h=g$, should not project out any state. Hence, at least the self-projection condition should be modified to

$$
V_{g} \cdot P_{\mathrm{sh}}-v_{g} \cdot\left(p_{\mathrm{sh}}+\Delta \tilde{N}_{g}\right) \equiv \frac{1}{2}\left(V_{g}^{2}-v_{g}^{2}+\frac{1}{4} B_{a} \cdot B_{3}\right)
$$

where $a=1$ for $g=g_{r^{\prime}}$ and $a=2$ for $g=g_{r}$. 


\subsection{Sample model as blow-up of orbifold with magnetized tori}

To illustrate our proposal we return to our example of an eight generation SU(5) GUT model discussed in subsection 4.4. Notice, that the bundle vectors $V_{r}$ defined in (4.22) can be interpreted as the shifted left-moving momenta $P_{\mathrm{sh}}$ of twisted states without oscillator excitations of a conventional $\mathbb{Z}_{2}$ orbifold, since $V_{r}^{2}=3 / 2$ is interpreted as the masslessness condition $P_{\mathrm{sh}}^{2}=3 / 2$. The bundle vectors $V_{r^{\prime}}^{\prime}$ on the other hand have ${V_{r^{\prime}}^{\prime}}^{2}=1 / 2$. In a conventional orbifold model these would correspond to twisted states with oscillators. However, as discussed in section 6, we expect that the left-moving mass formula gets modified to (6.3) in the presence of magnetized tori. If correct, one still interprets the $V_{r^{\prime}}^{\prime}$ as shifted left-moving momenta of twisted states without oscillators. Hence, even though this model has a blow-down limit, the resulting theory in this limit is not a conventional orbifold CFT.

Open Access. This article is distributed under the terms of the Creative Commons Attribution License which permits any use, distribution and reproduction in any medium, provided the original author(s) and source are credited.

\section{References}

[1] A.E. Faraggi, A new standard-like model in the four-dimensional free fermionic string formulation, Phys. Lett. B 278 (1992) 131 [INSPIRE].

[2] G. Cleaver, A. Faraggi and D.V. Nanopoulos, String derived MSSM and M-theory unification, Phys. Lett. B 455 (1999) 135 [hep-ph/9811427] [InSPIRE].

[3] T. Dijkstra, L. Huiszoon and A. Schellekens, Supersymmetric standard model spectra from RCFT orientifolds, Nucl. Phys. B 710 (2005) 3 [hep-th/0411129] [INSPIRE].

[4] T. Dijkstra, L. Huiszoon and A. Schellekens, Chiral supersymmetric standard model spectra from orientifolds of Gepner models, Phys. Lett. B 609 (2005) 408 [hep-th/0403196] [INSPIRE].

[5] L.J. Dixon, J.A. Harvey, C. Vafa and E. Witten, Strings on orbifolds, Nucl. Phys. B 261 (1985) 678 [inSPIRE].

[6] L.J. Dixon, J.A. Harvey, C. Vafa and E. Witten, Strings on orbifolds. 2, Nucl. Phys. B 274 (1986) 285 [inSPIRE].

[7] H.P. Nilles, S. Ramos-Sanchez, P.K. Vaudrevange and A. Wingerter, The orbifolder: a tool to study the low energy effective theory of heterotic orbifolds,

Comput. Phys. Commun. 183 (2012) 1363 [arXiv:1110.5229] [INSPIRE].

[8] O. Lebedev et al., A mini-landscape of exact MSSM spectra in heterotic orbifolds, Phys. Lett. B 645 (2007) 88 [hep-th/0611095] [INSPIRE].

[9] O. Lebedev, H.P. Nilles, S. Ramos-Sanchez, M. Ratz and P.K. Vaudrevange, Heterotic mini-landscape. (II) Completing the search for MSSM vacua in a Z(6) orbifold, Phys. Lett. B 668 (2008) 331 [arXiv:0807.4384] [INSPIRE].

[10] S. Nibbelink Groot, J. Held, F. Ruehle, M. Trapletti and P.K. Vaudrevange, Heterotic $Z(6-I I)$ MSSM orbifolds in blowup, JHEP 03 (2009) 005 [arXiv:0901.3059] [INSPIRE]. 
[11] W. Buchmüller, J. Louis, J. Schmidt and R. Valandro, Voisin-Borcea manifolds and heterotic orbifold models, JHEP 10 (2012) 114 [arXiv: 1208.0704] [INSPIRE].

[12] M. Blaszczyk et al., A $Z_{2} \times Z_{2}$ standard model, Phys. Lett. B 683 (2010) 340 [arXiv: 0911.4905] [INSPIRE].

[13] M. Blaszczyk, S. Nibbelink Groot, F. Ruehle, M. Trapletti and P.K. Vaudrevange, Heterotic MSSM on a resolved orbifold, JHEP 09 (2010) 065 [arXiv: 1007.0203] [INSPIRE].

[14] V. Bouchard and R. Donagi, An SU(5) heterotic standard model, Phys. Lett. B 633 (2006) 783 [hep-th/0512149] [INSPIRE].

[15] C. Schoen, On fiber products of rational elliptic surfaces with section, Math. Z. 197 (1988) 177.

[16] R. Donagi, B.A. Ovrut, T. Pantev and D. Waldram, Standard model bundles on nonsimply connected Calabi-Yau threefolds, JHEP 08 (2001) 053 [hep-th/0008008] [INSPIRE].

[17] R. Donagi, B.A. Ovrut, T. Pantev and D. Waldram, Spectral involutions on rational elliptic surfaces, Adv. Theor. Math. Phys. 5 (2002) 499 [math/0008011] [INSPIRE].

[18] R. Donagi, Y.-H. He, B.A. Ovrut and R. Reinbacher, The spectra of heterotic standard model vacua, JHEP 06 (2005) 070 [hep-th/0411156] [INSPIRE].

[19] T.L. Gomez, S. Lukic and I. Sols, Constraining the Kähler moduli in the heterotic standard model, Commun. Math. Phys. 276 (2007) 1 [hep-th/0512205] [INSPIRE].

[20] V. Braun, Y.-H. He, B.A. Ovrut and T. Pantev, A standard model from the $E_{8} \times E_{8}$ heterotic superstring, JHEP 06 (2005) 039 [hep-th/0502155] [INSPIRE].

[21] L.B. Anderson, J. Gray, A. Lukas and E. Palti, Two hundred heterotic standard models on smooth Calabi-Yau threefolds, Phys. Rev. D 84 (2011) 106005 [arXiv:1106.4804] [InSPIRE].

[22] L.B. Anderson, J. Gray, A. Lukas and E. Palti, Heterotic line bundle standard models, JHEP 06 (2012) 113 [arXiv:1202.1757] [INSPIRE].

[23] S. Donalson, Anti-self-dual Yang-Mills connections over complex algebraic surfaces and stable vector bundles, Proc. London Math. Soc. 50 (1985) 1.

[24] K. Uhlenbeck and S. Yau, On the existence of Hermitian-Yang-Mills connections in stable vector bundles, Comm. Pure Appl. Math. 19 (1986) 257.

[25] R. Blumenhagen, G. Honecker and T. Weigand, Supersymmetric (non-)abelian bundles in the type I and SO(32) heterotic string, JHEP 08 (2005) 009 [hep-th/0507041] [INSPIRE].

[26] R. Blumenhagen, G. Honecker and T. Weigand, Loop-corrected compactifications of the heterotic string with line bundles, JHEP 06 (2005) 020 [hep-th/0504232] [INSPIRE].

[27] G. Honecker and M. Trapletti, Merging heterotic orbifolds and K3 compactifications with line bundles, JHEP 01 (2007) 051 [hep-th/0612030] [INSPIRE].

[28] G. Honecker, Orbifolds versus smooth heterotic compactifications, arXiv:0709.2037 [INSPIRE].

[29] R. Donagi and K. Wendland, On orbifolds and free fermion constructions, J. Geom. Phys. 59 (2009) 942 [arXiv:0809.0330] [INSPIRE].

[30] A. Hebecker and M. Trapletti, Gauge unification in highly anisotropic string compactifications, Nucl. Phys. B 713 (2005) 173 [hep-th/0411131] [INSPIRE]. 
[31] R. Blumenhagen and E. Plauschinn, Intersecting D-branes on shift $Z_{2} \times Z_{2}$ orientifolds, JHEP 08 (2006) 031 [hep-th/0604033] [INSPIRE].

[32] M. Fischer, M. Ratz, J. Torrado and P.K. Vaudrevange, Classification of symmetric toroidal orbifolds, JHEP 01 (2013) 084 [arXiv:1209.3906] [INSPIRE].

[33] S.J. Konopka, Non abelian orbifold compactifications of the heterotic string, arXiv: 1210.5040 [INSPIRE].

[34] D. Cremades, L. Ibáñez and F. Marchesano, Computing Yukawa couplings from magnetized extra dimensions, JHEP 05 (2004) 079 [hep-th/0404229] [INSPIRE].

[35] H. Abe, K.-S. Choi, T. Kobayashi and H. Ohki, Non-abelian discrete flavor symmetries from magnetized/intersecting brane models, Nucl. Phys. B 820 (2009) 317 [arXiv:0904.2631] [INSPIRE].

[36] H. Abe, K.-S. Choi, T. Kobayashi and H. Ohki, Magnetic flux, Wilson line and orbifold, Phys. Rev. D 80 (2009) 126006 [arXiv:0907.5274] [InSPIRE].

[37] V. Bouchard and R. Donagi, On a class of non-simply connected Calabi-Yau threefolds, Commun. Num. Theor. Phys. 2 (2008) 1 [arXiv:0704.3096] [INSPIRE].

[38] F. Denef, M.R. Douglas and B. Florea, Building a better racetrack, JHEP 06 (2004) 034 [hep-th/0404257] [INSPIRE].

[39] D. Lüst, S. Reffert, E. Scheidegger and S. Stieberger, Resolved toroidal orbifolds and their orientifolds, Adv. Theor. Math. Phys. 12 (2008) 67 [hep-th/0609014] [INSPIRE].

[40] S. Groot Nibbelink, M. Trapletti and M. Walter, Resolutions of $C^{n} / Z_{n}$ orbifolds, their $\mathrm{U}(1)$ bundles and applications to string model building, JHEP 03 (2007) 035 [hep-th/0701227] [INSPIRE].

[41] S. Nibbelink Groot, T.-W. Ha and M. Trapletti, Toric resolutions of heterotic orbifolds, Phys. Rev. D 77 (2008) 026002 [arXiv:0707.1597] [INSPIRE].

[42] S. Nibbelink Groot, D. Klevers, F. Ploger, M. Trapletti and P.K. Vaudrevange, Compact heterotic orbifolds in blow-up, JHEP 04 (2008) 060 [arXiv: 0802 . 2809] [INSPIRE].

[43] S. Nibbelink Groot, Heterotic orbifold resolutions as $(2,0)$ gauged linear $\sigma$-models, Fortsch. Phys. 59 (2011) 454 [arXiv: 1012.3350] [INSPIRE].

[44] G. Aldazabal, A. Font, L.E. Ibáñez, A. Uranga and G. Violero, Nonperturbative heterotic $D=6, D=4, N=1$ orbifold vacua, Nucl. Phys. B 519 (1998) 239 [hep-th/9706158] [INSPIRE]. 